\title{
Antibody-drug conjugates plus Janus kinase inhibitors enable MHC-mismatched allogeneic hematopoietic stem cell transplantation
}

\author{
Stephen P. Persaud, ${ }^{1}$ Julie K. Ritchey, ${ }^{2}$ Sena Kim, ${ }^{2}$ Sora Lim, ${ }^{2}$ Peter G. Ruminski, ${ }^{2}$ Matthew L. Cooper, ${ }^{2}$ Michael P. Rettig, ${ }^{2}$ \\ Jaebok Choi, ${ }^{2}$ and John F. DiPersio² \\ 'Division of Laboratory and Genomic Medicine, Department of Pathology and Immunology and 2Division of Oncology, Department of Medicine, Washington University School of Medicine, \\ St. Louis, Missouri, USA.
}

\begin{abstract}
Despite the curative potential of hematopoietic stem cell transplantation (HSCT), conditioning-associated toxicities preclude broader clinical application. Antibody-drug conjugates (ADCs) provide an attractive approach to HSCT conditioning that minimizes toxicity while retaining efficacy. Initial studies of ADC conditioning have largely focused on syngeneic HSCT. However, to treat acute leukemias or induce tolerance for solid organ transplantation, this approach must be expanded to allogeneic HSCT (allo-HSCT). Using murine allo-HSCT models, we show that pharmacologic Janus kinase 1/2 (JAK1/2) inhibition combined with CD45- or cKit-targeted ADCs enables robust multilineage alloengraftment. Strikingly, myeloid lineage donor chimerism exceeding $99 \%$ was achievable in fully MHC-mismatched HSCT using this approach. Mechanistic studies using the JAK1/2 inhibitor baricitinib revealed marked impairment of T and NK cell survival, proliferation, and effector function. NK cells were exquisitely sensitive to JAK1/2 inhibition due to interference with IL-15 signaling. Unlike irradiated mice, ADC-conditioned mice did not develop pathogenic graft-versus-host alloreactivity when challenged with mismatched T cells. Finally, the combination of ADCs and baricitinib balanced graft-versus-host disease and graft-versus-leukemia responses in delayed donor lymphocyte infusion models. Our allo-HSCT conditioning strategy exemplifies the promise of immunotherapy to improve the safety of HSCT for treating hematologic diseases.
\end{abstract}

\section{Introduction}

Hematopoietic stem cell transplantation (HSCT) has therapeutic potential for hematologic malignancies, autoimmunity, immunodeficiency, chronic infection, or tolerance induction for solid organ transplantation (SOT) (1-3). However, two formidable barriers must be overcome to achieve successful HSCT outcomes. First, recipient-derived hematopoietic stem cells (HSCs) must be depleted to create space for incoming donor HSCs. Second, in allogeneic HSCT (allo-HSCT), host and donor immune responses must be controlled to prevent graft rejection and graftversus-host disease (GvHD), respectively (4). To overcome these barriers, HSCT patients undergo chemotherapy- and irradiation-based conditioning regimens (5), whose toxicities generally limit the use of HSCT to life-threatening conditions, such as acute myeloid leukemia (AML) (6).

\footnotetext{
Conflict of interest: JC declares consultancy with Daewoong Pharmaceutical and research support from Mallinckrodt Pharmaceuticals and Incyte Corporation. MLC declares consultancy, ownership equity, and royalties from Wugen, royalties from NeolmmuneTech, and consultancy with Rivervest. JFD declares consultancy with Rivervest, research support from Macrogenics, BioLine, NeolmmuneTech, and Incyte Corporation, and ownership equity with Magenta Therapeutics and Wugen. SPP and JFD have filed a patent application for the combination of antibody-drug conjugates with JAK inhibitors for HSCT conditioning (USPTO application 17/504,656).

Copyright: (C) 2021, American Society for Clinical Investigation.

Submitted: October 27, 2020; Accepted: October 27, 2021; Published: December 15, 2021.

Reference information: / Clin Invest. 2021;131(24):e145501.

https://doi.org/10.1172/JCl145501.
}

For AML, allo-HSCT offers the best chance for disease control. Donor T lymphocytes in the HSC allograft mediate graftversus-leukemia (GvL) effects that protect against relapse (7). Myeloablative conditioning is preferable for AML, as its antileukemia activity also mitigates relapse risk (8). However, since the median age at diagnosis for AML is 68 (9), patients' medical comorbidities or functional status may prevent them from undergoing this potentially curative therapy (10). Moreover, older AML patients have cytogenetically and clinically higher-risk disease that is more treatment resistant and relapse prone (11-13). This presents a clinical dilemma: the patients most likely to suffer from AML with adverse features are those who most require aggressive therapy, yet they are often the least able to tolerate it.

Allo-HSCT conditioning approaches that avoid treatmentrelated toxicities without sacrificing therapeutic efficacy are urgently needed. Recently, conditioning strategies have emerged using antibody-drug conjugates (ADCs) to make marrow space for HSCT. Initial studies used saporin-conjugated ADCs recognizing the phosphatase CD45 (CD45-SAP) or the tyrosine kinase c-Kit (cKit-SAP) to specifically ablate the hematopoietic niche (14, 15). In mouse models, CD45-SAP and cKit-SAP were well tolerated and effectively permitted syngeneic HSCT with multilineage donor chimerism. Moreover, these agents were used therapeutically in models of sickle cell disease (14), hemophilia (16), Fanconi anemia (17), and recombinase-activating gene (RAG) deficiency (18). Fewer studies, however, have considered ADCs as conditioning for allo-HSCT, in which T cell- and/or NK cell-mediated 
rejection must be overcome to enable engraftment $(19,20)$. Such studies are critical for applying ADC-based conditioning to AML or for tolerance induction in SOT.

Herein, we used minor histocompatibility antigen- (miHA-) and MHC-mismatched models to develop minimally toxic conditioning regimens for murine allo-HSCT and investigate how these therapies affect host and donor immunity. We demonstrate that selective Janus kinase $1 / 2$ (JAK1/2) inhibitors, previously shown to mitigate GvHD while preserving GvL effects (21), overcame the barriers to alloengraftment when combined with ADC-based conditioning. This strategy was particularly effective when ADCs were combined with an orally formulated JAK1/2 inhibitor, achieving fully MHC-mismatched HSCT with myeloid lineage donor chimerism exceeding 99\%. Unlike total body irradiation-based (TBIbased) conditioning, ADC-based conditioning did not promote pathogenic graft-versus-host alloreactivity in F1 mice challenged with parental splenocytes. Finally, using a donor lymphocyte infusion (DLI) model to study GvL responses, we show that CD45-SAP and JAK1/2 inhibition struck a therapeutic balance between tumor control and GvHD compared with mice receiving either treatment alone. Our study provides a strategy for allo-HSCT whose biological effects - preventing rejection while balancing GvHD and GvL responses - provide the ideal blend of immunomodulatory activities for the treatment of AML.

\section{Results}

Verification of CD45 and c-Kit ADCs for syngeneic HSCT conditioning. To evaluate CD45-SAP and cKit-SAP as conditioning agents for allo-HSCT, we compared their previously described abilities to promote syngeneic HSCT $(14,15)$. In vitro, CD45-SAP and cKit-SAP inhibited hematopoietic colony formation with picomolar-range $\mathrm{IC}_{50}$ values (Figure $1 \mathrm{~A}$ ). Both ADCs effectively depleted HSCs in vivo, as defined phenotypically (CD48-CD150 ${ }^{+}$ LSK) or by colony formation (Figure 1B). Importantly, HSC depletion required an intact $\mathrm{ADC}$ comprising the relevant antibody linked to saporin; controls lacking either of these components were devoid of activity. As previously reported, CD45-SAP was strongly lymphodepleting, whereas cKit-SAP lacked this activity (Supplemental Figure 1A; supplemental material available online with this article; https://doi.org/10.1172/JCI145501DS1). Notably, reduced CD8 ${ }^{+} \mathrm{T}$ cells and increased granulocytes were consistently observed in control mice receiving streptavidin-saporin (sAV-SAP) or IgG-SAP (Supplemental Figure 1A). These effects were more pronounced in the CD45-SAP studies and in the $50 \mu \mathrm{g}$ cKit-SAP group compared with the $10 \mu \mathrm{g}$ cKit-SAP group, possibly reflecting a dose-dependent effect of sAV-SAP itself. Finally, except for moderate thrombocytopenia in mice treated with $50 \mu \mathrm{g}$ cKit-SAP, complete blood counts (CBCs) remained within the reference range in all ADC-treated mice. (Supplemental Figure 1B).

In a syngeneic HSCT model with GFP-tagged B6 HSCs transplanted to WT B6 recipients (B6-GFP $\rightarrow$ B6), $75 \mu$ g CD45-SAP was well tolerated and permitted stable, high-level donor engraftment comparable to that reported previously (ref. 14 and Figure 1C). Although $10 \mu \mathrm{g}$ cKit-SAP depleted HSCs as effectively as $75 \mu \mathrm{g}$ CD45-SAP, it was somewhat less effective at promoting engraftment. However, when $50 \mu \mathrm{g}$ cKit-SAP was used, overall donor engraftment was equivalent to that seen with CD45-SAP. CBCs largely remained within reference limits during these experiments (Figure 1D), and donor chimerism in lymphoid organs mirrored that observed in peripheral blood (Supplemental Figure 2A). Finally, donor marrow from CD45-SAP- and cKit-SAP-conditioned primary transplant recipients was successfully transplanted into secondary recipients, confirming engraftment of functional HSCs (Figure 1E and Supplemental Figure 2B). Taken together, these studies confirm the efficacy of CD45-SAP and cKit-SAP as conditioning for HSCT in the absence of immunologic barriers.

CD45-SAP plus in vivo $T$ cell depletion enables engraftment in miHA- and MHC-mismatched allo-HSCT. To investigate the efficacy of ADCs for allo-HSCT conditioning, we used 2 transplant models (Figure 2A): an miHA-mismatched model (BALB/c-Ly5.1 $\rightarrow$ $\mathrm{DBA} / 2)$ and a haploidentical F1-to-parent $(\mathrm{CB} 6 \mathrm{~F} 1 \rightarrow \mathrm{B} 6)$ model mismatched for $\mathrm{H}-2^{\mathrm{d}}$ in the host-versus-graft direction. We focused on CD45-SAP for conditioning in these studies to leverage its lymphodepleting activity to overcome graft rejection. However, CD45-SAP alone failed to allow alloengraftment, likely due to its incomplete ablation of host $\mathrm{T}$ cells. This suggested further immunosuppression was needed to achieve alloengraftment.

We therefore treated CD45-SAP-conditioned animals with antibodies depleting $\mathrm{CD} 4^{+}$and/or $\mathrm{CD}^{+} \mathrm{T}$ cells throughout the peritransplant period (Figure 2A) and followed donor chimerism longitudinally (Supplemental Figure 3, A-C, and ref. 22). In the miHA model, in vivo $\mathrm{CD}^{+} \mathrm{T}$ cell depletion (TCD), but not $\mathrm{CD} 4^{+}$ TCD, was sufficient to produce engraftment in most (7/9) recipients. $\mathrm{CD}^{+}$and $\mathrm{CD}^{+}$pan-TCD of CD45-SAP-conditioned mice resulted in multilineage engraftment in all treated mice, albeit with significant variability in donor chimerism (Figure 2B). Gradual loss of donor chimerism was noted in 1 of 10 pan-TCD mice, with the shorter-lived myeloid cells showing the most rapid decline, a pattern suggesting failure of long-term HSC persistence. Low-level donor $\mathrm{T}$ cell engraftment was observed in pan-TCD mice conditioned with an inactive ADC. Finally, serial transplantation studies using marrow from CD45-SAP-conditioned, pan-TCD recipients confirmed engraftment of functional donor-derived HSCs (Supplemental Figure 3D). In the F1-toparent model, pan-TCD was required for engraftment (Figure 2C). High-level donor B cell and myeloid lineage chimerism and lower $\mathrm{T}$ cell chimerism were routinely observed in this system. Although all pan-TCD animals showed donor engraftment initially, 5 of 9 mice showed evidence of graft loss, with one showing a sudden, multilineage loss of donor-derived cells indicative of rejection. Serial CBCs in both models remained largely stable within reference limits (Figure 2, D and E).

Although the miHA model has potential for bidirectional alloreactivity, we observed neither overt graft rejection nor GvHD, suggesting achievement of stable mixed chimerism. To directly test for allotolerance, we surgically grafted BALB/c or DBA/2 skin into BALB-DBA mixed chimeras (Supplemental Figure 4A). Whereas DBA/2 mice that failed to engraft BALB/c HSCs rejected $\mathrm{BALB} / \mathrm{c}$ skin postoperatively, BALB-DBA chimeras tolerated both $\mathrm{BALB} / \mathrm{c}$ and DBA/2 skin grafts. As a secondary test, we adoptively transferred CFSE-labeled T cells from BALB-DBA chimeras to new cohorts of BALB/c, DBA/2, or CB6F1 mice (Supplemental Figure $4 \mathrm{~B})$. Ninety percent of the transferred T cells were Ly5.1 ${ }^{+}$ (derived from BALB/c-CD45.1 donors) and did not proliferate 
A

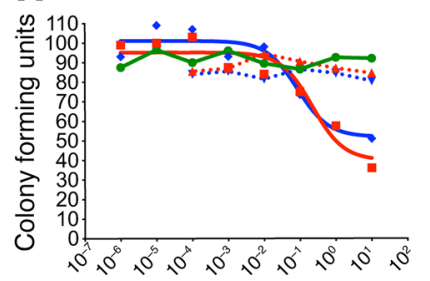

[Antibody-drug conjugate] (nM)

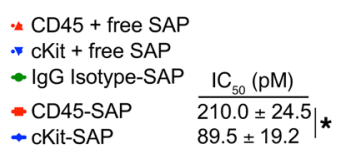

B

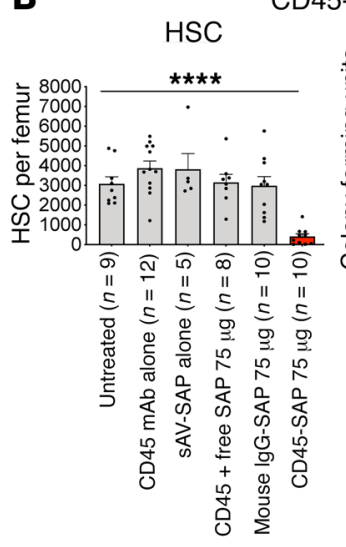

CD45-SAP

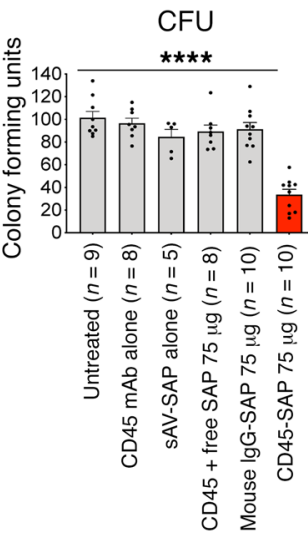

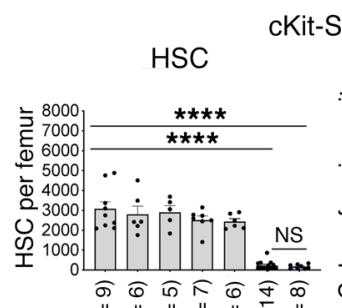

II II II II II II I

ఫ

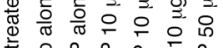

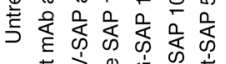

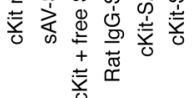

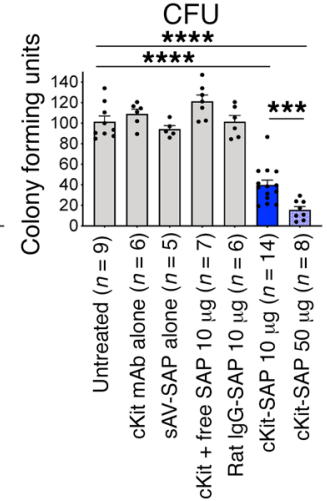

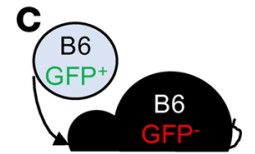
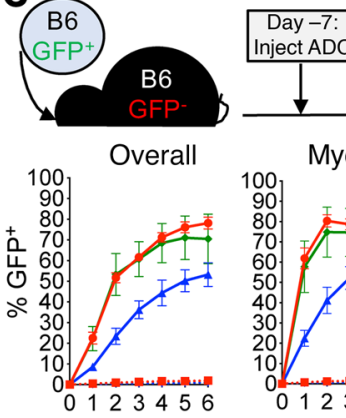

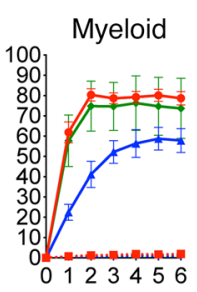

Months after transplant
- CD45-SAP $(n=8)$

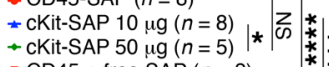
- CD45 + free SAP $(n=8)$ - cKit + free SAP $10 \mu \mathrm{g}(n=9)$

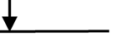

B cells

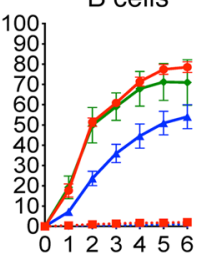

ant

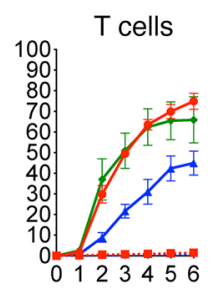

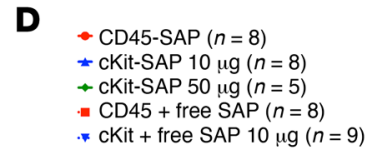
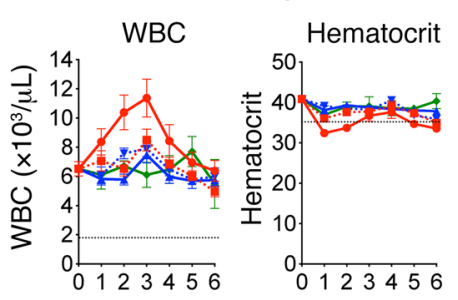

Months after transplant

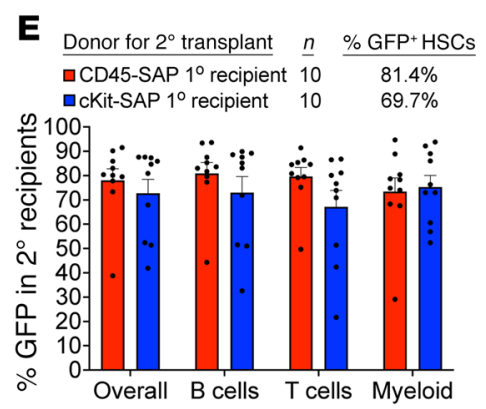

Figure 1. CD45-SAP and cKit-SAP are similarly effective as conditioning agents for syngeneic HSCT. (A) Inhibition of B6 bone marrow colony formation in vitro by $A D C s$ or control conjugates. Mean colony counts (from duplicate plates) from 1 representative of 3 experiments are shown. (B) In vivo depletion of bone marrow CD150+CD48- LSK cells (HSC) and CFUs 7 days after infusion with the indicated conjugates. Mice were pooled from 2 to 4 experiments; the same cohort of untreated mice was used to compare with the CD45-SAP and cKit-SAP treatment groups. (C and D) Schematic and results for syngeneic HSCT in mice conditioned with the indicated conjugates. Donor chimerism overall and by lineage (C) and CBCs (D) are displayed and were pooled from 2 to 3 experiments. (E) Secondary HSCT using whole marrow from B6-GFP $\rightarrow$ B6 primary recipients that were conditioned with the indicated ADCs, analyzed at 4 months after HSCT. The percentage of GFP+ ${ }^{+} \mathrm{HSCs}$ (donor-derived HSCs from primary recipients) infused to the secondary recipients is shown; mice were pooled from 2 experiments. Data points and error bars represent mean \pm SEM. Student's $t$ test (B, CD45-SAP), 1-way ANOVA (B, cKit-SAP), and repeated measures ANOVA (C) were used for statistical comparisons. ${ }^{*} P<0.05 ;{ }^{* * *} P<0.001 ;{ }^{* * *} P<0.0001$.

when infused into either BALB/c or DBA/2 mice. However, these cells proliferated robustly upon infusion into CB6F1 mice heterozygous for the foreign $\mathrm{H}-2^{\mathrm{b}}$ haplotype. Taken together, these results verify that our mixed chimeras developed cross-tolerance to donor and recipient tissue.

CD45-SAP combined with the JAK1/2 inhibitor baricitinib promotes multilineage engraftment in allo-HSCT recipients without in vivo TCD. Our studies using in vivo TCD in miHA- and MHC-mismatched allo-HSCT provide proof-of-principle evidence that ADC-based conditioning regimens can permit engraftment, provided that immune barriers are sufficiently suppressed. However, the variability of donor chimerism we observed, the high incidence of graft loss, and the potential risk of opportunistic infections would limit the clinical utility and translatability of a strategy requiring prolonged TCD. We therefore sought to refine our ADC-based allo-HSCT conditioning regimens with these issues in mind.

Prior work from our laboratory demonstrated that the selective JAK1/2 inhibitor baricitinib prevents and even reverses established GvHD, while preserving GvL effects (21). The complete 


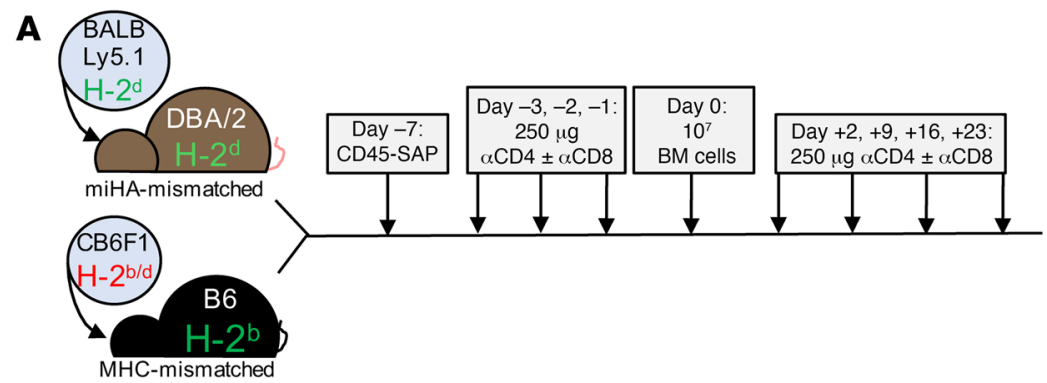

B

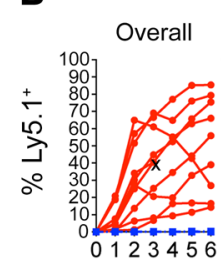

miHA-mismatched

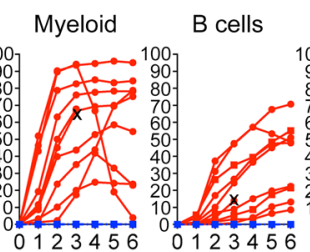

T cells

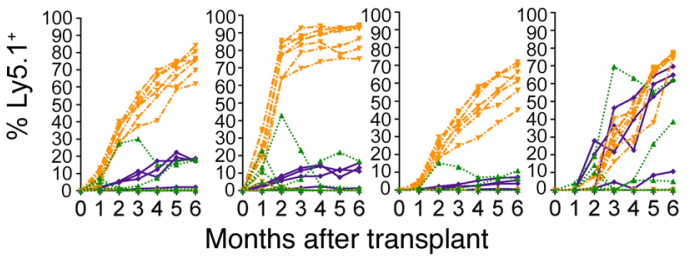

c
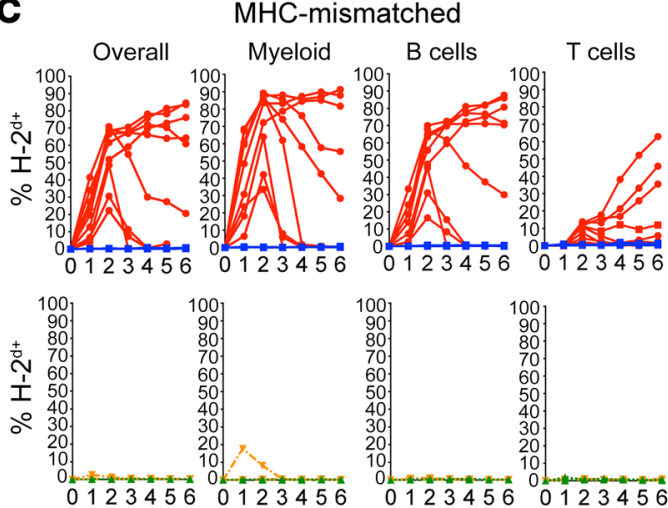

Months after transplant
D

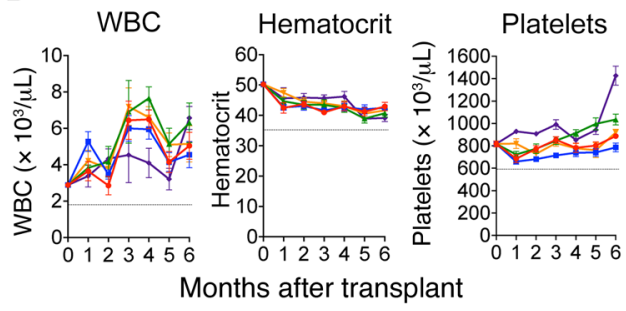

$\mathbf{E}$

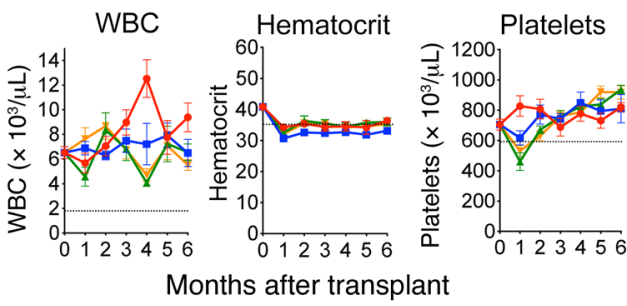

Figure 2. $\alpha \beta$ TCD in CD45-SAP-conditioned mice permits engraftment in miHA- and MHC-mismatched allo-HSCT. (A) Schematic for miHA- and MHCmismatched allo-HSCT models utilizing CD45-SAP plus TCD. (B and C) Peripheral blood donor chimerism for individual mice in the miHA-mismatched (B) and MHC-mismatched allo-HSCT models (C) pooled from 2 to 3 experiments. (D and E) Serial CBCs for miHA- (D) and MHC-mismatched (E) models.

$X$ indicates mouse euthanized for severe head tilt unrelated to the experimental treatment. Data points and error bars represent mean \pm SEM. Repeated measures ANOVA or mixed effects model (for group with missing data due to mouse loss) was used for statistical comparisons of overall donor chimerism. ${ }^{* *} P<0.01$; ${ }^{* *} P<0.001$; ${ }^{* * *} P<0.0001$.

prevention of GvHD seen with baricitinib phenocopied that seen in IFN- $\gamma \mathrm{R}$-deficient mice treated with $\alpha \mathrm{IL}-6 \mathrm{R}$, implicating these cytokines' signaling pathways as important targets of baricitinib's effects. Interestingly, mice that received baricitinib also showed somewhat improved donor chimerism, although this was in lethally irradiated mice with donor chimerism already near $100 \%$. However, in fully mismatched allo-HSCT utilizing sublethal irradiation to model reduced-intensity conditioning (RIC) (Supplemental Figure 5), IFN- $\gamma \mathrm{R}$ deficiency in donor and/or recipient cells markedly improved donor chimerism. This result suggested that genetically disabling IFN- $\gamma \mathrm{R}$ signaling could permit engraftment in the context of an RIC regimen and that a JAK1/2 inhibitor, having as one of its several downstream effects the pharmacologic interference with IFN- $\gamma \mathrm{R}$ signaling, might prove similarly effective in the context of ADC conditioning.

Consequently, we tested baricitinib in our allo-HSCT models, first using it in lieu of TCD (Figure 3, A and B). CD45-SAP conditioning plus daily baricitinib was highly effective in the miHA-mismatched model, with most mice (7/10) stably engrafting with approximately $80 \%$ overall donor chimerism. However, daily baricitinib treatment plus CD45-SAP was not effective in promoting engraftment in the MHC-mismatched model. Engraftment was also not observed in MHC-mismatched HSCT when CD45-SAP was combined with posttransplant cyclophosphamide 
A

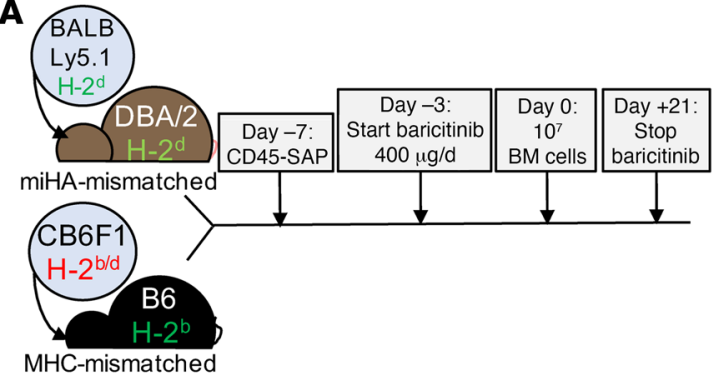

MHC-mismatched

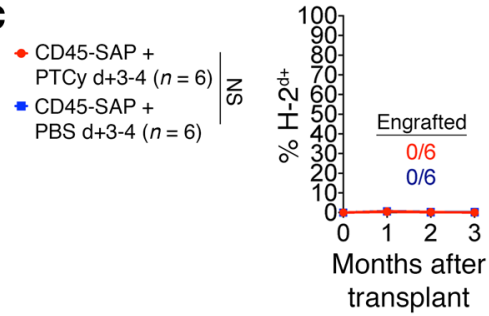

B miHA-mismatched * CD45-SAP +

* $\quad$ baricitinib $(n=10)$

- CD45-SAP +
vehicle $(n=10)$

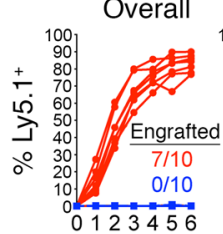

Myeloid

B cells

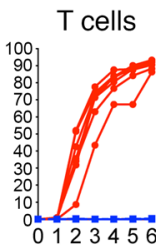

MHC-mismatched

- CD45-SAP +

0 baricitinib $(n=7)$

$=$ CD45-SAP +

vehicle $(n=5)$
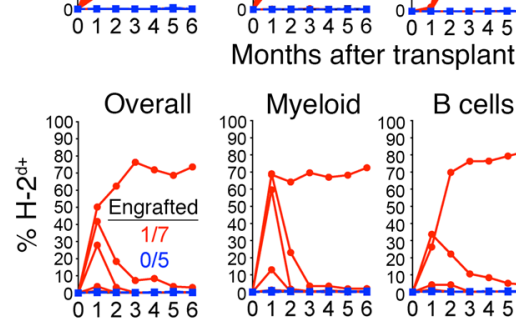

Months after transplant

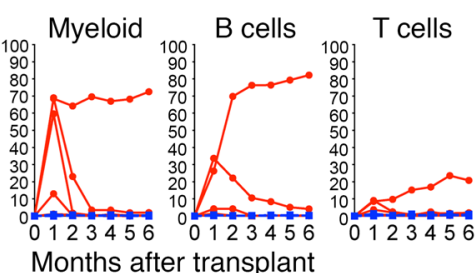

D
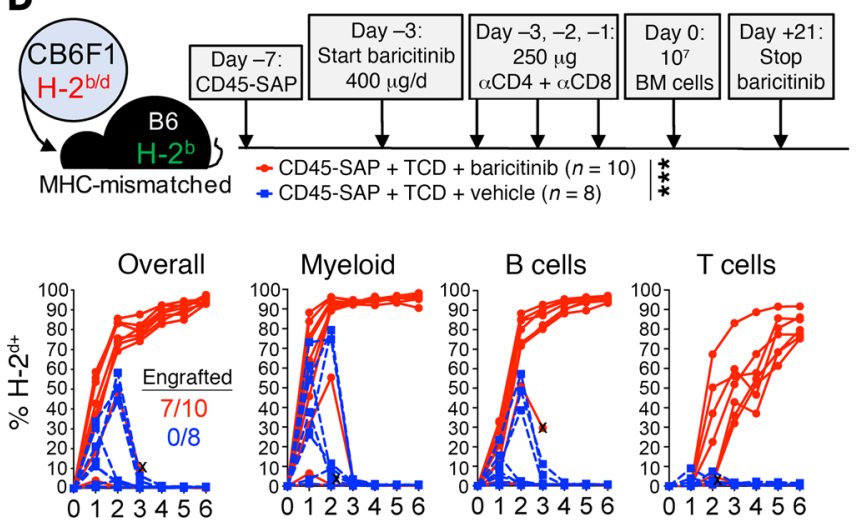

Months after transplant
$\mathbf{E}$
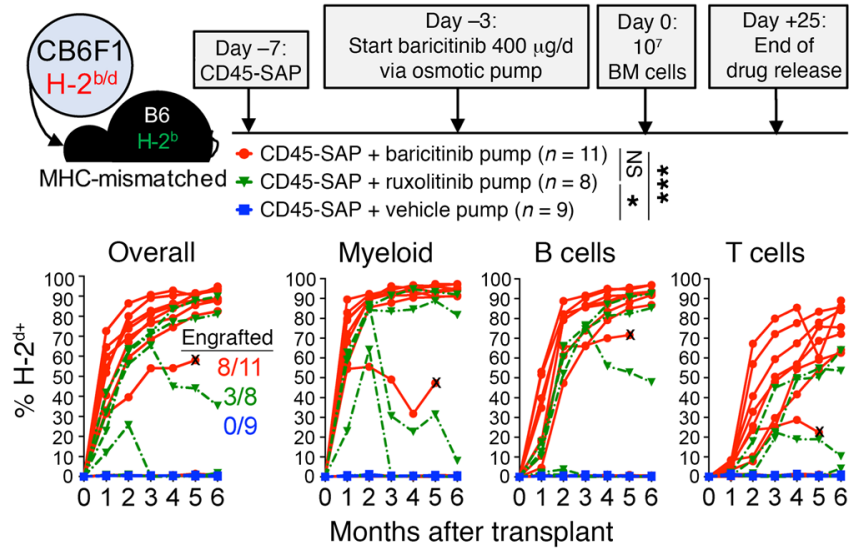

Figure 3. The selective JAK1/2 inhibitor baricitinib permits alloengraftment in CD45-SAP-conditioned mice. (A) Schematic for baricitinib and CD45-SAP treatment in allo-HSCT models. (B) Peripheral blood donor chimerism for individual mice in the miHA-mismatched and MHC-mismatched models, pooled from 2 experiments. (C) Overall donor chimerism in MHC-mismatched HSCT combining CD45-SAP with PTCy or PBS, pooled from 2 experiments. (D) Schematic and results for MHC-mismatched HSCT combining CD45-SAP, daily baricitinib, and pretransplant TCD, pooled from 3 experiments. (E) Schematic and results for MHC-mismatched HSCT combining CD45-SAP with continuously infused JAK1/2 inhibitors, pooled from 3 experiments. X indicates mouse death or euthanasia. Insets display the proportion of successfully engrafted mice at $t=6$ months. Repeated measures ANOVA (B and C) and a mixed effects model ( $\mathbf{D}$ and $\mathbf{E}$ ) were used for statistical comparisons of overall donor chimerism. ${ }^{*} P<0.05$; ${ }^{* *} P<0.001$.

(PTCy), a standard-of-care immunosuppressant used as GvHD prophylaxis in human allo-HSCT (Figure 3C and ref. 23).

Since T cells make up the major barrier to engraftment in our MHC-mismatched model (Figure 2), failed engraftment in mice receiving daily baricitinib likely reflects insufficient suppression of these cells. To address this, we combined CD45-SAP and daily baricitinib therapy with pretransplant pan-TCD, essentially substituting baricitinib for posttransplant TCD (Figure 3D). This regimen was highly effective, achieving stable engraftment in 7 of 10 mice, with overall donor chimerism greater than $90 \%$. The donor chimerism in all lineages, particularly $\mathrm{T}$ cells, was superior to that seen with baricitinib or pan-TCD alone. In contrast, vehicle-treated mice experienced graft loss by 3 months after HSCT. Thus, posttransplant immunosuppression with baricitinib effectively preserves graft stability when combined with pretransplant lymphodepletion.
We next considered pharmacological explanations for the poor efficacy of daily baricitinib monotherapy in MHC-mismatched HSCT. Data from a prior study showed that subcutaneous baricitinib has a plasma half-life in $\mathrm{B} 6$ mice of approximately 1 hour (24), suggesting a prolonged absence of circulating drug if dosed every 24 hours. To test the duration of the baricitinib effect, we conducted a pharmacodynamic (PD) study in which mice received a single baricitinib dose, then were followed over time with a whole-blood assay for IFN- $\gamma$-induced Stat1 phosphorylation. Baricitinib at $400 \mu \mathrm{g}$ strongly suppressed Stat1 phosphorylation through 24 hours after infusion and became ineffective by 36 hours. In comparison, $80 \mu \mathrm{g}$ baricitinib provided only partial suppression at 4 hours after infusion that was absent thereafter (Supplemental Figure 6A). The related JAK1/2 inhibitor ruxolitinib partially inhibited Stat1 phosphorylation at 4 hours after infusion only at the $400 \mu \mathrm{g}$ dose and became ineffective by 12 
hours (Supplemental Figure 6B). Notably, pan-TCD did not affect baricitinib efficacy (Supplemental Figure 6C), making this an unlikely contributor to the improved engraftment when pan-TCD and baricitinib were used together (Figure 3D).

We hypothesized that a continuous presence of baricitinib would provide more sustained immunosuppression. We therefore administered the same $400 \mu \mathrm{g}$ daily dose of baricitinib continuously via subcutaneous osmotic pumps. Baricitinib in a $50 \%$ DMSO/50\% PEG-400 vehicle was compatible with delivery via osmotic pumps and retained bioactivity both in vitro after prolonged incubation at $37^{\circ} \mathrm{C}$ and in vivo (Supplemental Figure 7). Continuously infused baricitinib (Figure $3 \mathrm{E}$ ) was more effective than daily baricitinib (Figure 3B) in promoting multilineage engraftment in MHC-mismatched allo-HSCT, achieving more than $80 \%$ overall donor chimerism in most (8/11) mice. Continuously infused ruxolitinib at the same dose permitted engraftment in fewer mice than baricitinib, consistent with our PD studies of the 2 compounds. As we consistently observed in the MHC-mismatched model recipients dosed daily with JAK inhibitors, mice with baricitinib pumps developed a mild anemia in the first month after HSCT that corrected at subsequent time points; otherwise, $\mathrm{CBCs}$ remained within reference limits (Supplemental Figure 8).

Taken together, these studies demonstrate multiple effective, feasible strategies using CD45-SAP and JAK1/2 inhibitors to achieve high-level donor chimerism in both miHA- and MHC-mismatched allo-HSCT without requiring prolonged, global T cell ablation.

Baricitinib promotes engraftment via suppression of T cell-and NK cell-mediated rejection. We next pursued the mechanisms by which baricitinib promotes engraftment in allo-HSCT. While suppression of $\mathrm{T}$ cell alloreactivity is likely an important component, disruption of JAK1/2 signaling may affect engraftment in other ways, such as direct effects on donor hematopoiesis (25-27). To investigate the degree to which immunosuppression versus other mechanisms contributes to engraftment, we applied baricitinib to CD45-SAPconditioned syngeneic HSCT, in which immune barriers to engraftment are absent. In peripheral blood and lymphoid organs (Figure 4, A and B), no significant difference in donor chimerism was observed between CD45-SAP-conditioned mice receiving baricitinib versus vehicle. Importantly, no engraftment was observed in baricitinib-treated mice conditioned with inactive ADC, indicating that baricitinib alone cannot enable donor HSC engraftment.

To characterize the acute effects of baricitinib treatment on HSCT recipients, we analyzed peripheral blood and lymphoid organs of $\mathrm{B} 6$ mice that received 4 daily doses of baricitinib, the same number of doses given before HSCT. Baricitinib treatment minimally affected CBCs or bone marrow cellularity, but was associated with a significant reduction in spleen cellularity (Supplemental Figure 9, A and B). Bone marrow hematopoietic stem and progenitor cells (HSPCs) were unaffected by baricitinib except for showing somewhat lower frequencies of long-term HSC and megakaryocyte-erythroid progenitors (Supplemental Figure 9C). Mature hematopoietic cell counts were also minimally affected except for a mild reduction in splenic B cells and a marked depletion of peripheral NK cells (Supplemental Figure 9, D-F). Finally, immunophenotyping of the splenic $\mathrm{T}$ cell and antigen-presenting cell (APC) compartments revealed no differences between baricitinib- and vehicle-treated mice (Supplemental Figure 9, G and H).
We next cultured anti-CD3-stimulated, CFSE-labeled B6 T cells with baricitinib in vitro. Baricitinib impaired $\mathrm{CD} 4^{+}$and $\mathrm{CD} 8^{+}$ $\mathrm{T}$ cell expansion in a dose-dependent manner (Figure 4C) due to increased cell death and mildly reduced cell proliferation (Figure 4D). As expected with primary murine cells, unstimulated cultures showed significant $\mathrm{T}$ cell death after 72 hours. Importantly, the degree of cell death in these cultures was only subtly increased by baricitinib at the highest tested dose, arguing against nonspecific toxicity. Concentrations of TNF- $\alpha$, IL- 6 , and particularly IFN- $\gamma$ in the culture supernatants were significantly reduced by baricitinib (Figure 4E). This reduction in cytokine secretion was not generalizable, as IL-2 secretion was unaffected by baricitinib. In summary, while baricitinib minimally perturbed resting $\mathrm{T}$ cells, activated $\mathrm{T}$ cell function was more adversely affected. This is consistent with our hypothesis that baricitinib acts predominantly via immunosuppression, exerting its major therapeutic function on alloreactive T cells that respond to donor HSCs.

Extending baricitinib-based conditioning to fully haploidentical (F1 $\rightarrow$ F1) and fully MHC-mismatched models (BALB/c $\rightarrow$ B6) requires inhibition of both $\mathrm{T}$ and NK cells. We noted that baricitinib strongly depleted peripheral NK cells (Supplemental Figure 9D), which has been shown previously for ruxolitinib in mice and humans (28-30). We therefore tested to determine whether baricitinib could protect against NK cell-mediated rejection using a parent-to-F1 $(\mathrm{B} 6 \rightarrow \mathrm{CB} 6 \mathrm{~F} 1)$ allo-HSCT model (Figure $5 \mathrm{~A})$. In this setting, engraftment of parental HSCs is resisted by CB6F1 NK cells responding to the absence of $\mathrm{H}-2^{\mathrm{d}}$ on the donor-derived cells ("missing self" recognition; ref. 31). This phenomenon, also termed hybrid resistance, provides an opportunity to isolate NK cell-mediated host-versus-graft responses and investigate how baricitinib affects them.

Overall donor chimerism in $\mathrm{B} 6 \rightarrow \mathrm{CB} 6 \mathrm{~F} 1$ transplants treated with CD45-SAP plus vehicle was approximately 25\% 4 months after HSCT (Figure 5A), considerably lower than that obtained in syngeneic HSCT (Figure 1C). Engraftment was improved to a similar degree by both $\alpha$ NK1.1 depletion and treatment with baricitinib. Pre-HSCT analysis of peripheral blood confirmed that both $\alpha$ NK1.1 and baricitinib treatment markedly depleted CB6F1 recipients' circulating NK cells (Figure 5B). Thus, baricitinib overcame NK cellmediated barriers to HSCT due to efficient in vivo NK cell depletion.

NK cell development, maturation, and function depend upon IL-15, which signals through JAK1 and JAK3 to activate Stat5 (32). We asked whether baricitinib disrupts this critical signaling pathway to compromise NK cell survival and function. Murine NK cells stimulated in vitro with IL-15 alone or a cocktail of IL-12, IL-15, and IL-18 (33) showed dose-dependent increases in cell death and decreases in IFN- $\gamma$ production in response to baricitinib (Figure 5C). As with T cells, nonspecific toxicity in unstimulated cultures was modest and noted only at the highest baricitinib dose. In longer cultures, baricitinib impaired IL-15-mediated NK cell expansion, an effect attributable to dramatically reduced proliferation and viability (Figure 5D). Baricitinib also strongly suppressed IL-15-induced upregulation of the cytolytic enzymes perforin and granzyme B (Figure $5 \mathrm{E}$ ), which are required for full NK cell cytotoxicity (34). However, baricitinib did not prevent killing of YAC-1 target cells by NK cells that were already primed with IL-15, suggesting that baricitinib inhibits the acquisition but not 

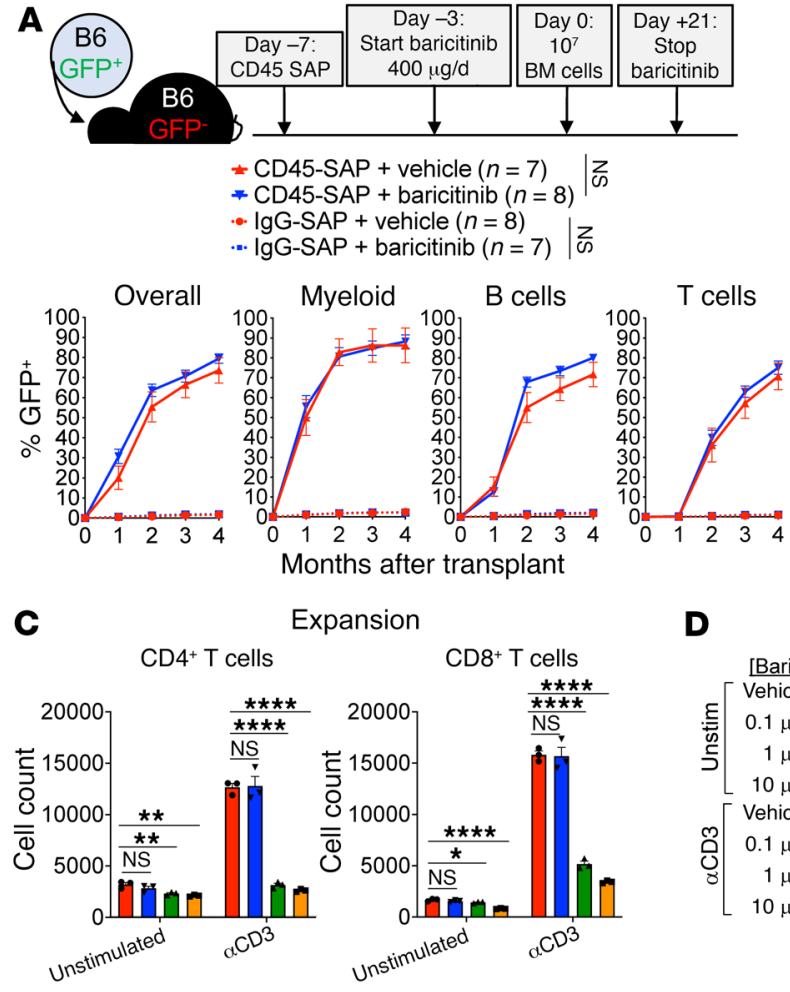

B
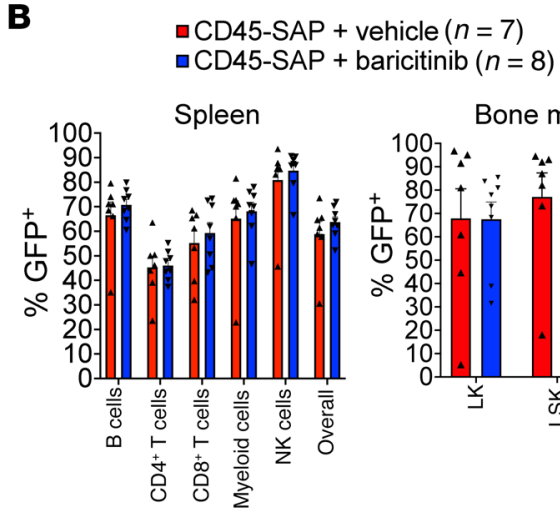
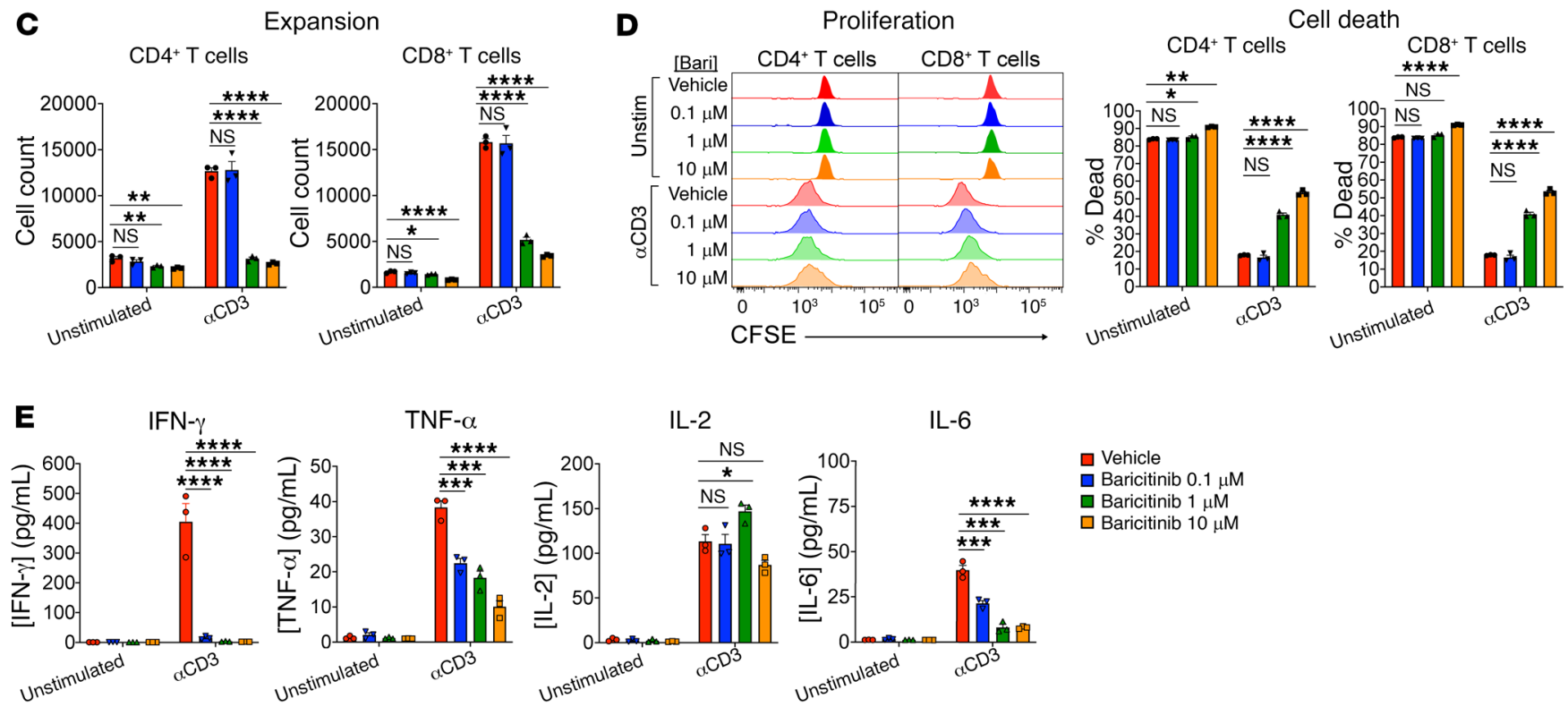

Figure 4. Baricitinib suppresses T cell function and viability and minimally affects syngeneic HSCT. (A) Schematic and results for baricitinib and CD45SAP treatment in syngeneic HSCT, pooled from 2 experiments. (B) Donor chimerism in spleen and bone marrow of mice from A. (C) In vitro expansion of $\alpha \mathrm{CD} 3$-stimulated $(1 \mu \mathrm{g} / \mathrm{mL}, 72$ hours), CFSE-labeled B6 T cells in the presence of baricitinib. (D) Proliferation and viability of cultures in C. (E) Cytokines in supernatants of cultures from $\mathbf{C}$ after 24 hours incubation. For $\mathbf{C}-\mathbf{E}$, data from 3 technical replicates are shown from 1 representative of 4 experiments. Data points and error bars represent mean \pm SEM. Repeated measures ANOVA (A, overall donor chimerism) and 1-way ANOVA (C-E) were used for statistical comparisons. ${ }^{*} P<0.05$; ${ }^{* *} P<0.01$; ${ }^{* *} P<0.001$; ${ }^{* * *} P<0.0001$.

the execution of NK cytotoxicity (Figure 5F). Finally, phosphoflow cytometry confirmed that baricitinib inhibits IL-15-induced Stat5 phosphorylation in a dose-dependent manner (Figure 5G).

To assess the ability of baricitinib to inhibit NK cell function in vivo, we infused baricitinib- or vehicle-treated mice with polyinosinic:polycytidylic acid (Poly I:C), a synthetic dsRNA analog and TLR3 agonist that mimics the inflammatory responses to viral infection, including stimulation of NK cells $(34,35)$. Poly I:C treatment induced NK cell expression of IFN- $\gamma$, perforin, and granzyme B and promoted cytotoxicity against YAC-1 target cells (Supplemental Figure 10). All of these effector activities were severely inhibited in mice that were pretreated with baricitinib. Taken together, our studies demonstrate that baricitinib potently impairs NK cell viability, proliferation, and effector function via interference with the IL-15/Stat5 signaling axis.
Orally formulated ruxolitinib plus ADC-based conditioning enables robust, high-level engraftment in fully MHC-mismatched $H S C T$. That baricitinib can inhibit both $\mathrm{T}$ and NK cell responses suggests it should be able to overcome the barrier these cells impose to fully MHC-mismatched HSCT. Indeed, CD45-SAP plus continuously infused baricitinib permitted high-level donor engraftment of BALB/c marrow into B6 mice (Supplemental Figure 11A), but in under half (3/7) of the recipients. Achieving engraftment in all experimental animals has proven elusive in our experiments thus far. This issue, coupled with the invasiveness of daily injections and, especially, osmotic pump implantation, prompted us to further optimize our conditioning method to improve robustness and efficacy while minimizing animal stress and trauma. We therefore used mouse chow formulated with ruxolitinib ( $2 \mathrm{~g}$ drug $/ \mathrm{kg}$ chow), thereby leveraging the high oral bioavailability of JAK1/2 inhibi- 
A

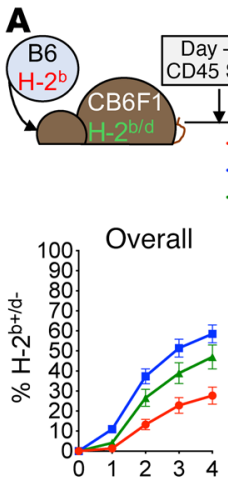

C

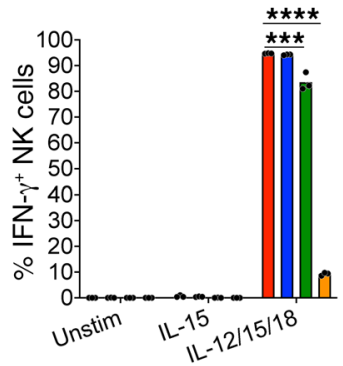

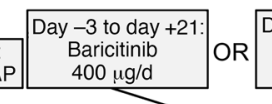

Day $-2,-1,0,+2,+4$ $+7,+10$, and +14 . $+7,+10$, and $+14:$
$250 \mu \mathrm{g} \alpha \mathrm{NK} 1.1$$\quad \begin{gathered}10^{7} \\ \mathrm{BM} \text { cells }\end{gathered}$

- CD45-SAP + vehicle $(n=10) \quad$ | $n * \star \star \mid$

- CD45-SAP + baricitinib $\left.(n=10)\right|^{\star \star *}$ *

- CD45-SAP + aNK1.1 $(n=10) \mid$ NS
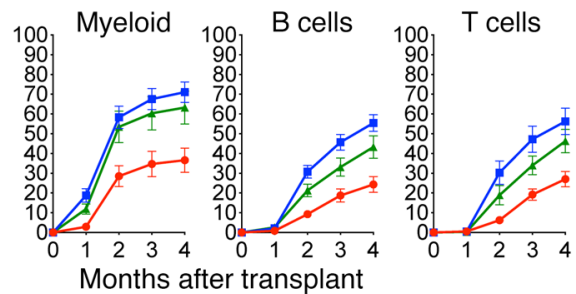
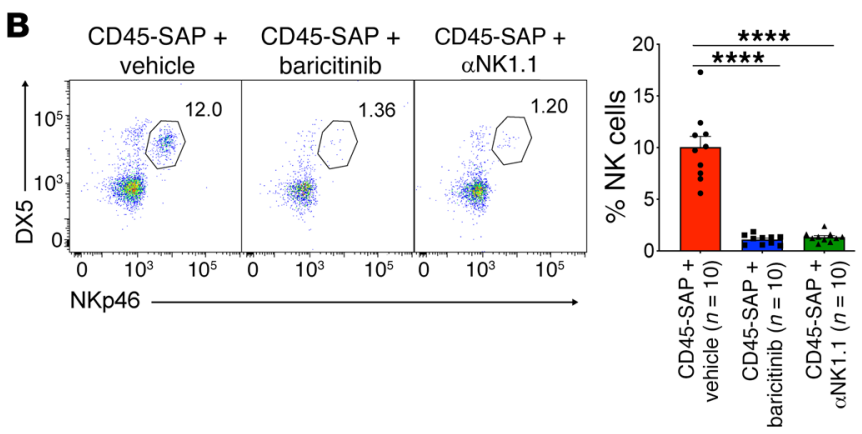
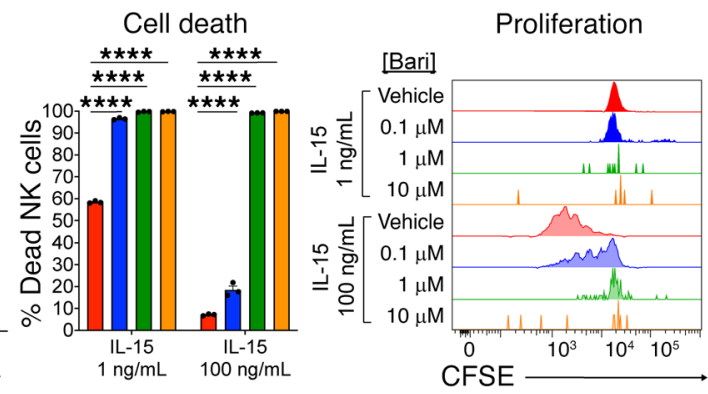

IL-15 $100 \mathrm{ng} / \mathrm{mL}$ (24 h)

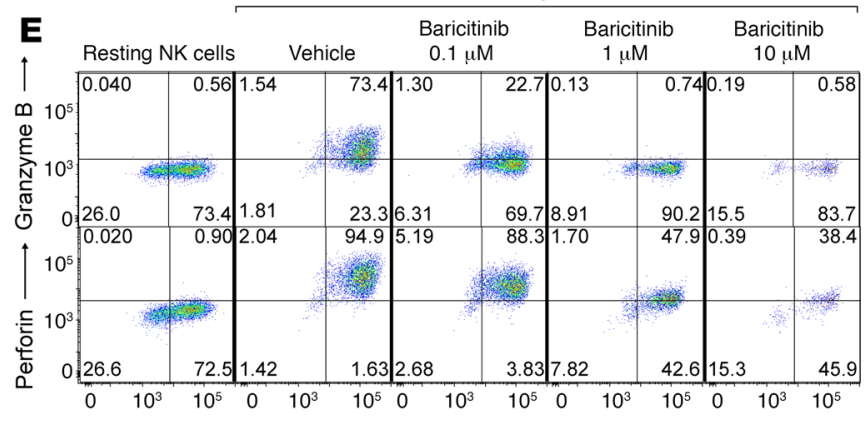

Granzyme A
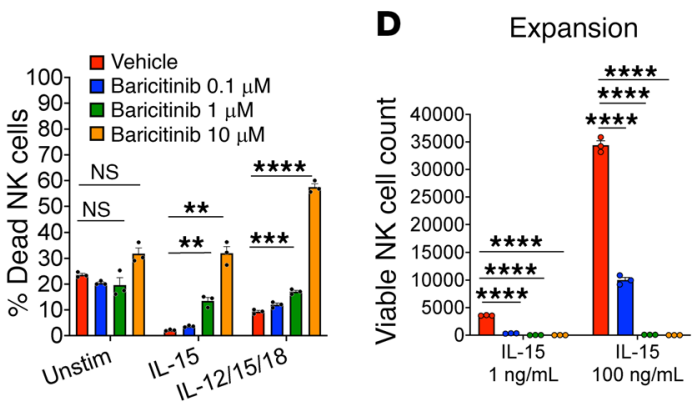

G

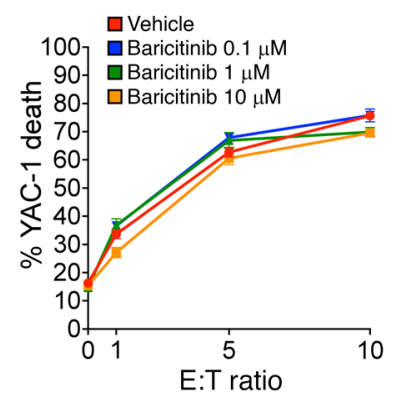

Figure 5. Baricitinib overcomes NK cell-mediated rejection by impairing NK cell survival and effector function. (A) Schematic and results for parent-to-F1 HSCT model to study baricitinib effects on NK cell-mediated rejection, pooled from 2 experiments. (B) Peripheral blood NK cell frequencies (insets) of recipients in A immediately before HSCT. (C-E) Functional assays of IL-15-stimulated B6 splenic NK cells incubated with baricitinib (3 technical replicates per condition): IFN- $\gamma$ production and survival after 15 hours (C), expansion and cell death after 72 hours (D), and cytolytic enzyme expression after 24 hours (E). (F) YAC-1 killing by splenic NK cells primed with IL-15 for 48 hours without baricitinib, then washed and plated with target cells for 4 hours with baricitinib. (C) NK cell phospho-Stat5 after IL-15 stimulation with or without baricitinib present. Inset numbers in $\mathbf{E}$ are cell frequencies within each quadrant. C-G show 1 representative of 3 experiments. Data points and error bars represent mean \pm SEM. Repeated measures ANOVA (A, overall donor chimerism) and 1-way ANOVA (B-D) were used for statistical comparisons. ${ }^{*} P<0.05 ;{ }^{* *} P<0.01 ;{ }^{* *} P<0.001 ;{ }^{* * *} P<0.0001$.

tors. Mice consume ruxolitinib chow as readily as normal chow, achieving steady-state drug concentrations comparable to that seen in humans (36-41). We tested whether this formulation could simplify JAK1/2 inhibitor administration while promoting donor engraftment with ADC-based conditioning.

Ruxolitinib chow combined with CD45-SAP was highly effective in promoting allo-HSCT (Figure 6, A and B), enabling stable engraftment in all recipients of miHA-mismatched grafts (Figure 6C) and most recipients (9/12) of fully MHC-mismatched grafts (Figure 6D). Of note, although 3 fully mismatched HSCT recipients experienced graft loss, all mice avoided early rejection and showed some initial evidence of engraftment. Overall, donor chi- merism in both models was comparable to that seen in prior studies, with most mice developing between $60 \%$ and $90 \%$ donor chimerism by 4 months after HSCT. While some variability in donor chimerism remained, particularly in the miHA-mismatched model, these results nevertheless have improved upon the engraftment success of our earlier experiments and successfully extended our strategy to the fully MHC-mismatched setting.

These promising findings led us to revisit the cKit-SAP ADC to test its efficacy in combination with ruxolitinib chow. Nearly all miHA-mismatched HSCT recipients engrafted with this regimen, with overall donor chimerism comparable to that seen with CD45-SAP (Figure 6E). Furthermore, in fully mismatched HSCT, 


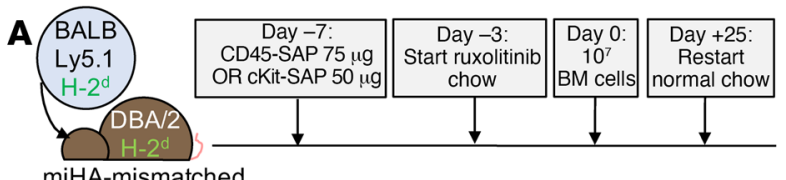

miHA-mismatched

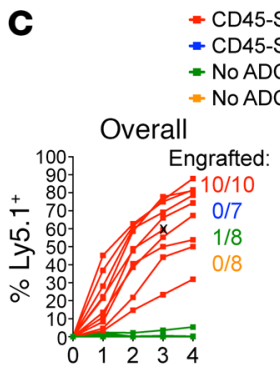

CD45-SAP + ruxolitinib chow $(n=10)\left|\begin{array}{l}* \\ * \\ *\end{array}\right| \begin{aligned} & * \\ & * \\ & * \\ & *\end{aligned}$

ADC + ruxolitinib chow $(n=8)$ Myeloid
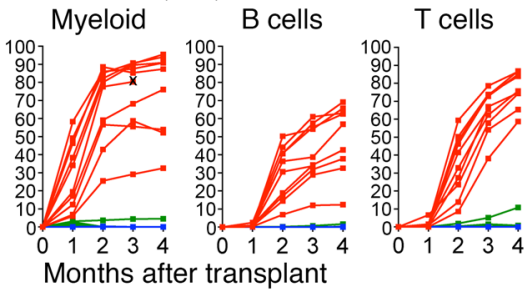

$\mathbf{E}$
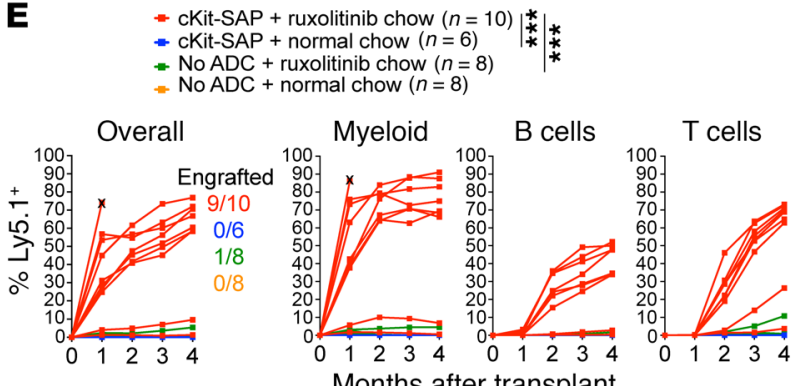

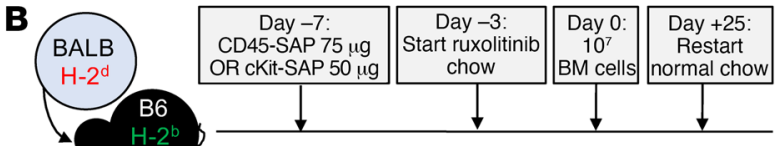

Fully MHC-mismatched
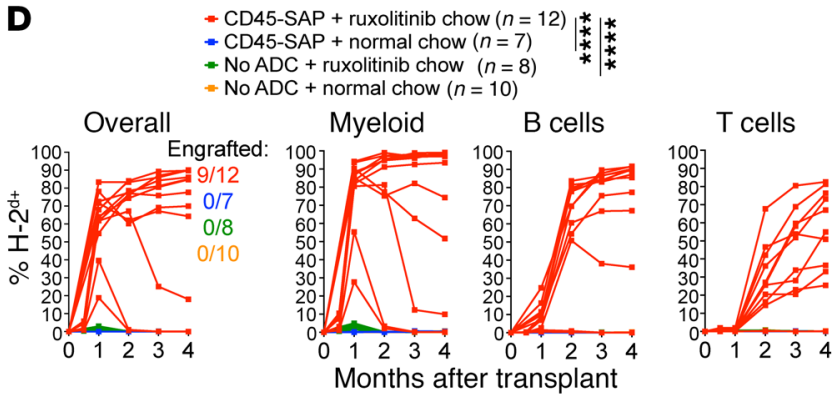

$\mathbf{F}$
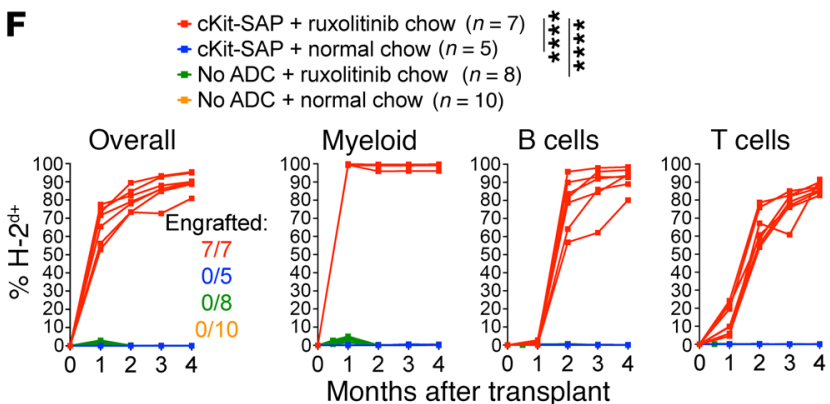

Figure 6. Orally formulated ruxolitinib plus CD45-SAP or cKit-SAP promotes robust, stable engraftment in miHA- and fully MHC-mismatched alloHSCT. (A and B) Schematic for miHA- (A) and fully MHC-mismatched (B) HSCT with ADC conditioning plus ruxolitinib chow. (C and D) Donor chimerism in individual miHA-mismatched (C) and fully mismatched (D) HSCT recipients conditioned with CD45-SAP plus ruxolitinib chow, pooled from 3 experiments. (E and F) Donor chimerism in individual miHA-mismatched (E) and fully mismatched (F) HSCT recipients conditioned with cKit-SAP plus ruxolitinib chow, pooled from 3 experiments. X indicates mouse death or euthanasia. Repeated measures ANOVA (C, D, F) and a mixed effects model (E) were used for statistical comparisons of overall donor chimerism. ${ }^{* *} P<0.001 ;{ }^{* * *} P<0.0001$.

every recipient engrafted with robust, high-level donor chimerism when cKit-SAP plus ruxolitinib chow was used (Figure 6F). Strikingly, myeloid lineage donor chimerism in these experiments was routinely $99 \%$ or greater by 1 month after HSCT. Thus, from our least to our most stringently mismatched models, CD45- and cKit-ADCs plus orally formulated JAK1/2 inhibitors permit robust donor engraftment with stable CBCs (Supplemental Figure 11B) via a simple, minimally invasive conditioning protocol.

ADC-based conditioning stimulates less pathogenic graft-versushost alloreactivity than TBI. The contribution of conditioning regimen intensity to the development of acute and chronic GvHD is well studied (42-45). A multitude of variables affect GvHD risk, including donor and recipient age, GvHD prophylaxis, donor HSC source and relatedness, and degree of human leukocyte antigen (HLA) disparity, which can influence the choice of conditioning intensity (46). Theoretically, host tissue injury caused by chemotherapy and radiation amplifies GvHD via release of endogenous damage- and pathogen-associated molecular patterns from dying cells. These mediators activate innate immunity, arming APCs to prime vigorous alloreactive $\mathrm{T}$ cell responses (47-49). We asked whether CD45-SAP and cKit-SAP, with their minimal tissue toxicities, would behave similarly.

To study the effect of conditioning regimen on $\mathrm{T}$ cell alloresponses in vivo, we used a parent-to-F1 adoptive transfer model, in which alloreactivity is exclusively in the graft-versus-host direction (Figure 7A). In this system, sublethally irradiated CB6F1 mice that receive $\mathrm{B} 6$ splenocytes develop pancytopenia secondary to $\mathrm{T}$ cell-mediated marrow aplasia (50). We compared CD45-SAP and cKit-SAP to sublethal rather than lethal irradiation (as is often used in standard GvHD models) to more closely match the conditioning regimens' severities. CD45-SAP, cKit-SAP, and 500 cGy irradiation are nonlethal and permit comparable levels of syngeneic engraftment $(14,15)$, suggesting a similar ability to generate marrow space for HSCT.

Compared with ADC-conditioned mice, TBI-conditioned mice infused with allogeneic splenocytes showed poorer clinical courses, including greater weight loss (Figure 7B). While mouse deaths most often occurred among TBI-conditioned mice, mortality was an inconsistent finding in this model. By 3 weeks after splenocyte infusion, TBI-conditioned, but not ADC-conditioned, mice developed pancytopenia (Figure 7C) and increased plasma concentrations of several inflammatory cytokines, particularly IFN- $\gamma$ (Figure 7D). Importantly, TBI-conditioned mice receiving syngeneic splenocytes and unconditioned mice receiving allogeneic splenocytes showed no evidence of disease, confirming that TBI plus allogeneic T cells are required for pathology. Circulating allogeneic donor $\mathrm{T}$ cells were present in ADC-conditioned mice albeit in low numbers, suggesting their lack of disease is not 

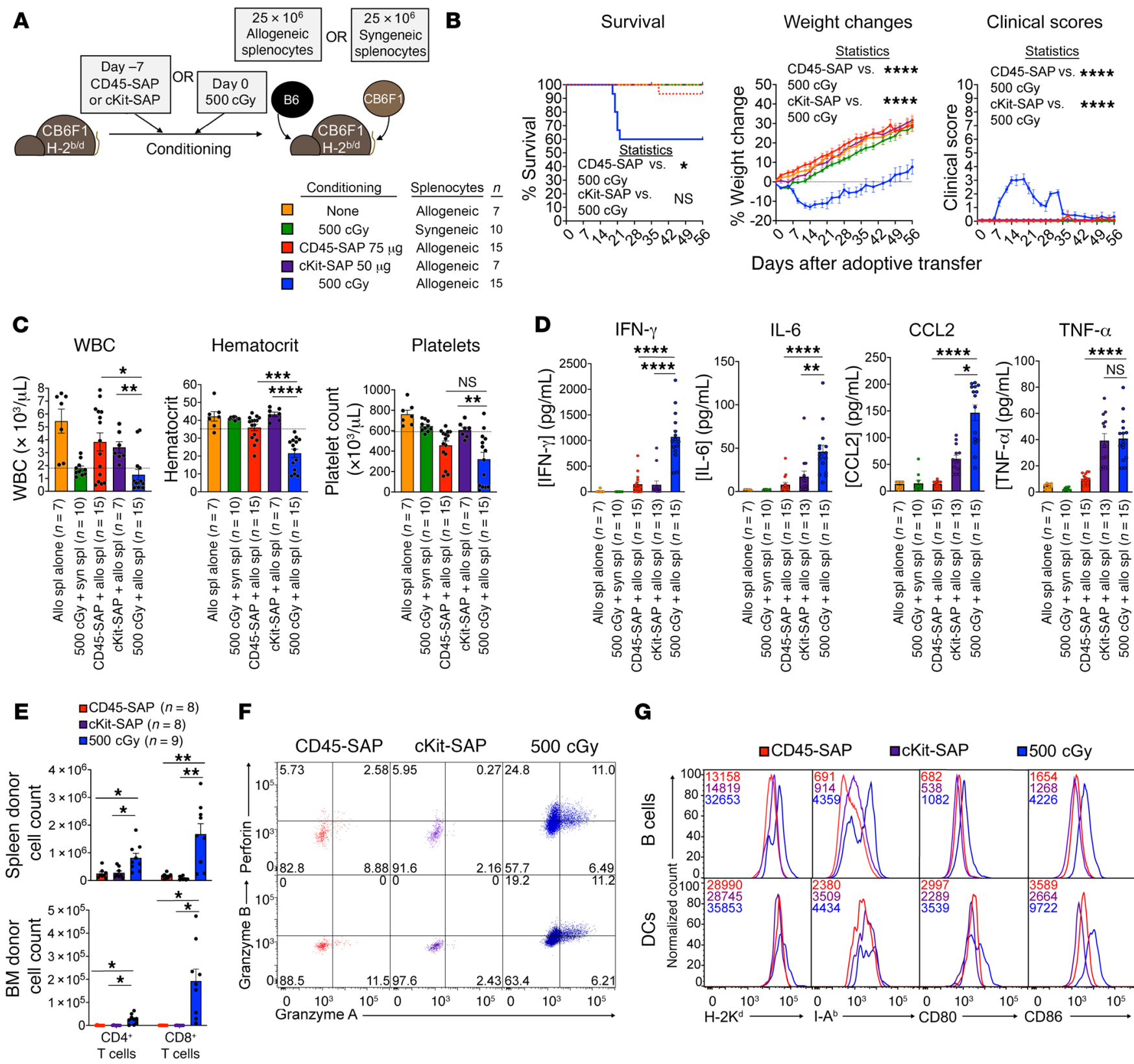

$\mathbf{F}$

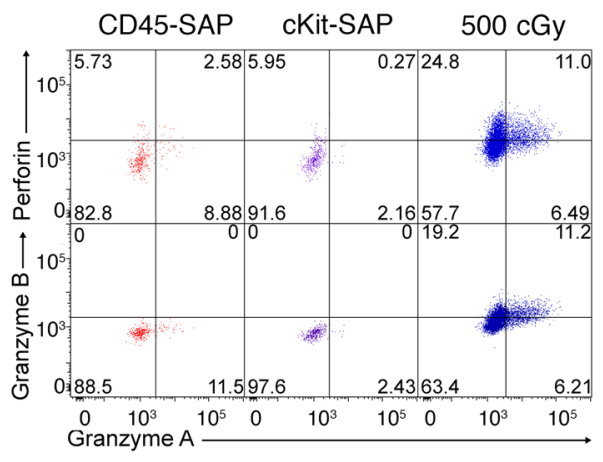

G

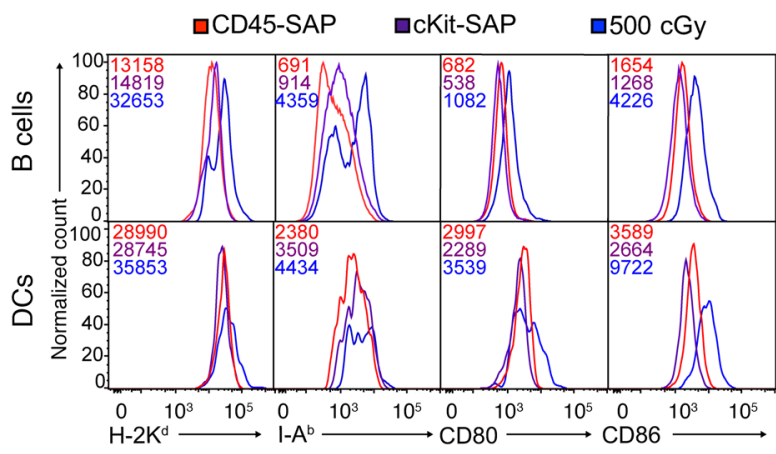

Figure 7. CD45-SAP and cKit-SAP conditioning do not promote graft-versus-host alloreactivity. (A) Schematic and legend for parent-to-F1 adoptive transfer model, with sublethal TBI or ADC conditioning. (B) Clinical outcomes for mice treated per A, pooled from 2 (cKit-SAP and no-conditioning groups) or 3 (all other groups) experiments; X indicates death or euthanasia. (C) CBCs at 3 weeks after splenocyte infusion. (D) Plasma inflammatory cytokine concentrations 7 days after splenocyte infusion. (E-G) Absolute donor $C D 4^{+}$and $C D 8^{+} T$ cell counts in spleen and bone marrow pooled from 3 experiments $(\mathbf{E})$, donor CD8+ $T$ cell cytolytic enzyme expression (F), and host APC phenotyping (G) 7 days after splenocyte infusion. In $\mathbf{F}$, inset numbers show cell frequencies within each quadrant; in G, inset numbers show MFIs. For $\mathbf{F}$ and $\mathbf{G}$, plots are from 1 representative mouse from 3 experiments. Data points and error bars represent mean \pm SEM. Mantel-Cox test (B, survival), mixed effects model (B, weights and clinical scores), 1-way ANOVA (C and E), and Kruskal-Wallis test (D) were used for statistical comparisons. ${ }^{*} P<0.05$; ${ }^{* *} P<0.01$; ${ }^{* *} P<0.001 ;{ }^{* * * *} P<0.0001$.

due to complete absence of donor T cells (Supplemental Figure 12A). Finally, bone marrow histopathology and flow cytometry demonstrated profound marrow aplasia and HSPC depletion in the TBI-conditioned mice that developed lethal disease (Supplemental Figure 12B).

To understand why allogeneic $\mathrm{T}$ cells failed to elicit disease in ADC-conditioned mice, we analyzed the early donor $\mathrm{T}$ cell response in ADC- versus TBI-conditioned mice. Donor $\mathrm{CD} 4^{+}$ and $\mathrm{CD} 8^{+} \mathrm{T}$ cells were identified in the spleens of both ADC- and TBI-conditioned mice, with greater total donor cell counts and somewhat higher frequencies of cells with an effector phenotype $\left(\mathrm{CD} 44^{\mathrm{hi}} \mathrm{CD} 62 \mathrm{~L}^{\mathrm{lo}}\right)$ in TBI-conditioned mice (Figure 7E and Supplemental Figure 12C). While the marrows of TBI-conditioned mice were extensively infiltrated by donor $\mathrm{T}$ cells, marrows of ADC-conditioned mice were virtually devoid of donor $\mathrm{T}$ cells. Expression differences in the bone marrow-homing chemokine 
receptor CXCR4 on donor $\mathrm{T}$ cells could not account for differences in marrow infiltration between ADC-and TBI-conditioned mice (Supplemental Figure 12C). Functionally, donor $\mathrm{CD}^{+} \mathrm{T}$ cells in irradiated mice notably upregulated perforin and granzymes A and B, indicating greater capacity for cytotoxicity than their counterparts in ADC-conditioned mice (Figure 7F). Higher expression of MHC and the costimulatory receptors CD80 and CD86 (Figure 7G) were observed in host-derived APCs, particularly B cells, from irradiated mice compared with ADC-conditioned mice. Collectively, these data suggest that ADC conditioning produces a suboptimal environment for priming a pathogenic graft-versus-host $\mathrm{T}$ cell alloresponse.

Comparison of baricitinib and cyclophosphamide effect on pathogenic graft-versus-host alloreactivity. Currently, ruxolitinib is approved for the treatment of steroid-refractory GvHD (51). However, its ability to treat or prevent GvHD while preserving GvL effects in preclinical models, taken together with its effectiveness at promoting engraftment, suggests a potential role for JAK1/2 inhibitors as GvHD prophylactic agents in the immediate peritransplant period (21). To further examine this possibility and to investigate the effect of baricitinib on $\mathrm{T}$ cell function in vivo, we tested the effect of baricitinib on graft-versus-host alloreactivity. Equivalent studies with PTCy were done to directly compare this established GvHD prophylactic agent with baricitinib.

Both baricitinib and PTCy prevented development of overt clinical disease in our parent-to-F1 model, with more rapid weight recovery following irradiation and splenocyte transfer compared with their respective vehicle controls (Figure 8A). Baricitinib-treated mice had a notably greater hematologic recovery at 3 weeks after splenocyte infusion than mice treated with PTCy (Figure $8 \mathrm{~B})$. Both baricitinib and PTCy reduced plasma CCL2 and TNF- $\alpha$ concentrations, but baricitinib was superior at reducing levels of IL- 6 and particularly IFN- $\gamma$ (Figure $8 \mathrm{C}$ ). In terms of donor T cell responses, baricitinib-treated mice had lower numbers of splenic and bone marrow $\mathrm{CD}^{+} \mathrm{T}$ cells, but unaffected total $\mathrm{CD} 4^{+} \mathrm{T}$ cell numbers (Figure 8D). PTCy, in contrast, effectively reduced splenic counts of donor $\mathrm{CD}^{+}$and, less consistently, $\mathrm{CD}^{+} \mathrm{T}$ cells. However, the marrows of PTCy-treated mice invariably showed minimal infiltration by donor $\mathrm{CD}^{+}$or $\mathrm{CD} 8^{+} \mathrm{T}$ cells. While host-derived (nonalloreactive) splenic $\mathrm{CD} 4^{+}$Treg counts were unaffected by baricitinib or PTCy, the alloreactive donor Treg compartment was expanded by baricitinib and depleted by PTCy. Phenotypically, the frequency of $\mathrm{CD} 44^{+} \mathrm{CD} 62 \mathrm{~L}^{-} \mathrm{CD} 4^{+}$donor $\mathrm{T}$ cells was modestly reduced in PTCy-treated mice; otherwise, donor T cell effector markers and CXCR4 expression were unaffected by either drug (Supplemental Figure 12D). Donor $\mathrm{CD}^{+} \mathrm{T}$ cell cytolytic enzyme expression was strongly inhibited by baricitinib; in contrast, splenic $\mathrm{CD}^{+} \mathrm{T}$ cells from PTCy-treated mice had somewhat increased frequencies of perforin- and granzyme-expressing cells (Figure 8E). Finally, host APC phenotyping of baricitinib- and PTCy-treated mice (Figure $8 \mathrm{~F}$ ) showed that both compounds mildly reduced MHC I and II expression by B cells, but only baricitinib downregulated class I and, particularly, class II on dendritic cells. Both drugs modestly downregulated CD86 on B cells with minimal effect on CD80; on dendritic cells, baricitinib reduced both CD80 and CD86 expression, whereas PTCy increased CD80 expression and minimally affected CD86.
In summary, baricitinib suppressed expansion and effector function of donor $\mathrm{CD}^{+} \mathrm{T}$ cells, promoted the expansion of splenic CD4 ${ }^{+}$Tregs, and inhibited APC function. PTCy, in contrast, strongly inhibited donor conventional $\mathrm{CD}^{+}$cell and Treg expansion, with variable effects on $\mathrm{CD} 8^{+} \mathrm{T}$ cells, including modestly enhanced cytolytic enzyme expression. However, bone marrow infiltration by donor T cells was absent in PTCy-treated mice. Overall, our studies confirm the previously described abilities of baricitinib and PTCy to mitigate pathogenic alloreactivity and highlight key similarities and differences in how they shape the donor alloresponse.

CD45-SAP plus baricitinib balances GvHD with protective $G v L$ responses. Finally, we asked how the combination of ADCs and JAK1/2 inhibitors would affect GvL responses. For these studies, we employed a well-characterized delayed DLI model (21) in which lethally irradiated BALB/c mice are engrafted with syngeneic, luciferase-expressing A20 lymphoma cells, then infused with donor lymphocytes 11 days after HSCT. CD45-SAP was used for these studies to test its ability to target the CD $45.2^{+}$A20 cells in vivo, as demonstrated in vitro (Figure 9A). For consistency, CD45SAP was given on $\mathrm{d}+4$ to maintain the 7-day interval between ADC infusion and adoptive transfer used in studies thus far (Figure 9B). Previous work by our group showed that baricitinib (200 or 400 $\mu \mathrm{g}, 5 \mathrm{~d} /$ wk starting $\mathrm{d}+12$ ) preserves the antitumor benefit provided by DLI, with a notable reduction in tumor burden after the drug was stopped (21). However, to prevent graft rejection and graftversus-host alloresponses in the present study, we used a more intensive baricitinib dosing regimen (400 $\mu \mathrm{g}$ baricitinib, $7 \mathrm{~d} / \mathrm{wk}$ starting 3 days before HSCT or splenocyte infusion) that may adversely affect tumor control. We therefore determined whether the more aggressive JAK1/2 inhibitor regimen used herein would affect GvL responses and how the addition of CD45-SAP would affect tumor control.

On pre-DLI imaging, CD45-SAP-treated mice showed consistently lower baseline tumor burden (Figure 9C), suggesting direct antitumor activity mediated by the ADC. However, as anticipated, a single CD45-SAP dose was insufficient to provide long-lasting tumor control in mice not receiving DLI. In mice receiving DLI, mice receiving vehicle with or without CD45-SAP showed similarly excellent tumor control, but also developed severe GvHD leading to near-complete mortality. GvHD secondary to DLI was potently inhibited by baricitinib, but baricitinib-treated mice had higher pre-DLI tumor burden that progressed despite DLI. However, adding CD45-SAP to baricitinib in DLI mice improved tumor control with only moderately worsened GvHD severity compared with that in baricitinib-only mice, leading to similar overall survival between the 2 groups. Thus, while the intensive baricitinib dosing used in this study impairs tumor control, this is counteracted by CD45-SAP without promoting severe GvHD, thus achieving a balance between GvHD and GvL activity that is absent in mice treated with CD45-SAP or baricitinib alone.

\section{Discussion}

Toxicities from chemotherapy- and radiation-based conditioning remain a major obstacle to the broader application of HSCT for the treatment of hematopoietic diseases, particularly for elderly or infirmed patients. Antibody-based HSCT conditioning presents a way to mitigate toxicities without compromising therapeutic effi- 

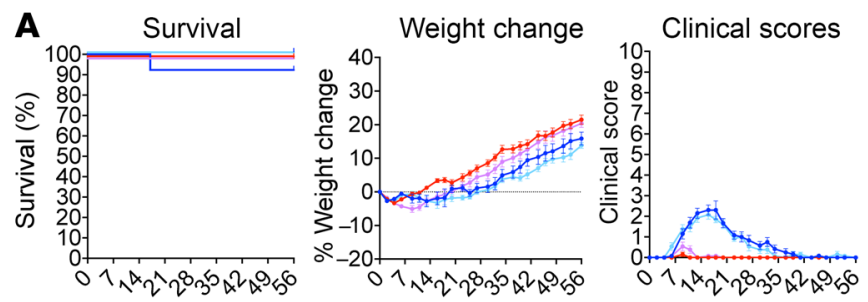

Days after adoptive transfer

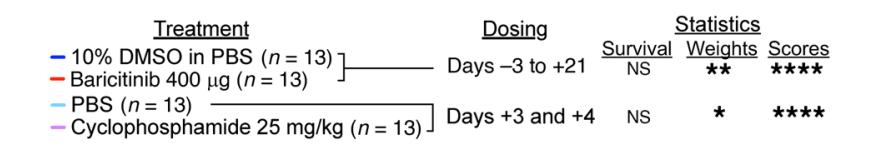

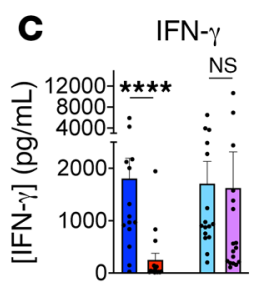

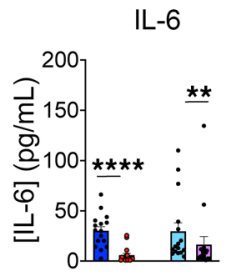

CCL2

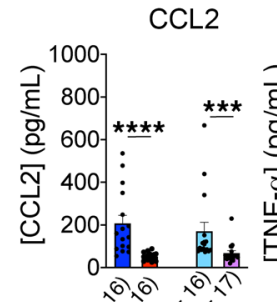

TNF- $\alpha$

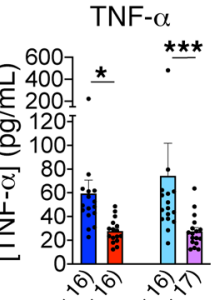

(6), 61,107
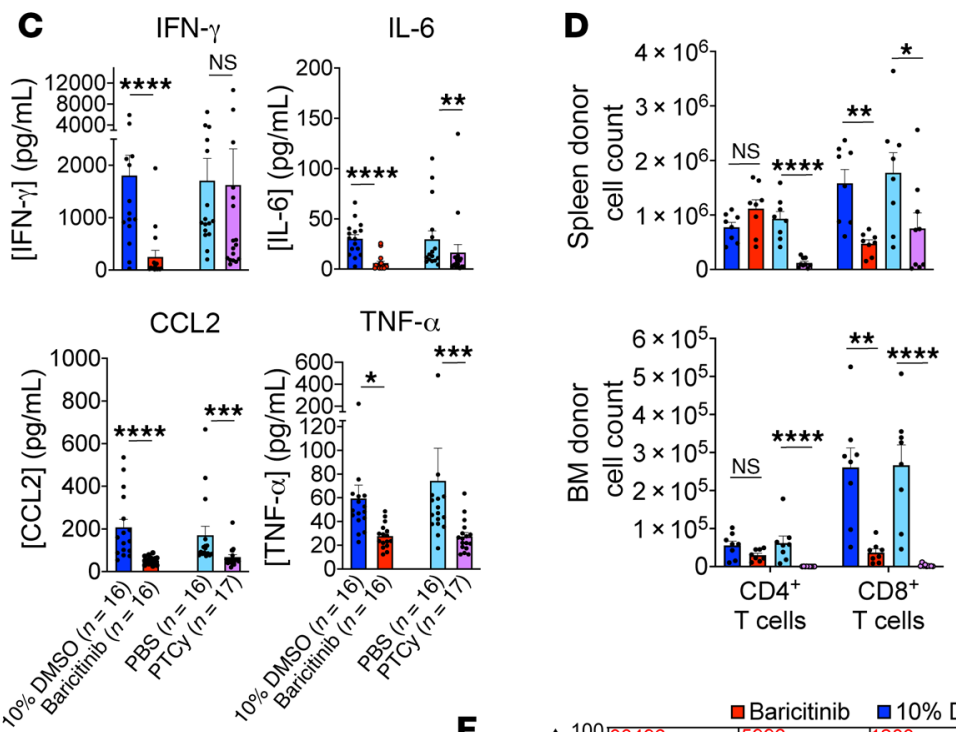
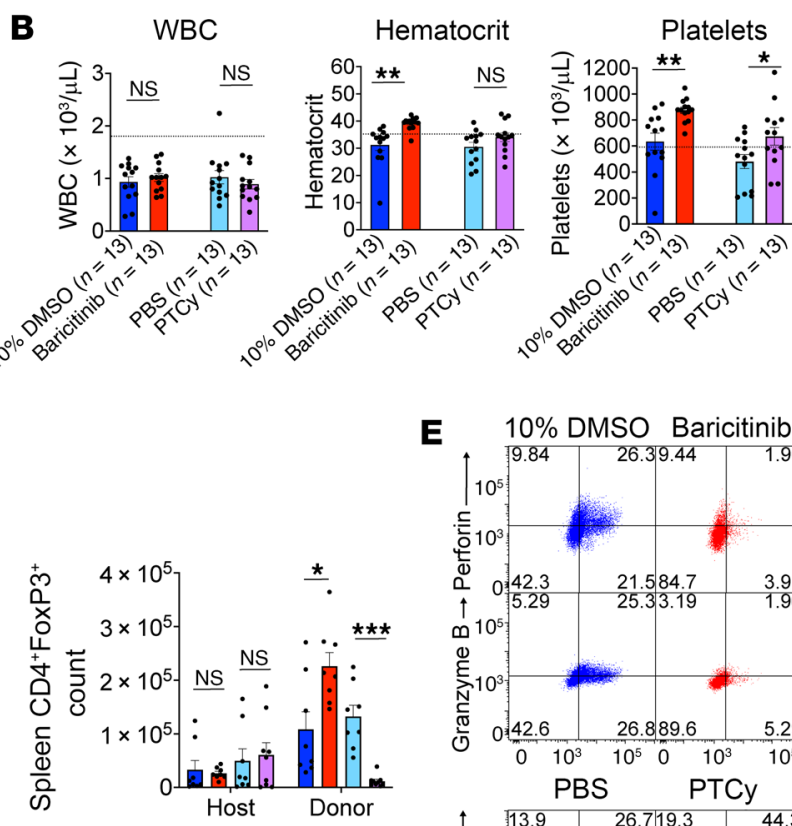

E
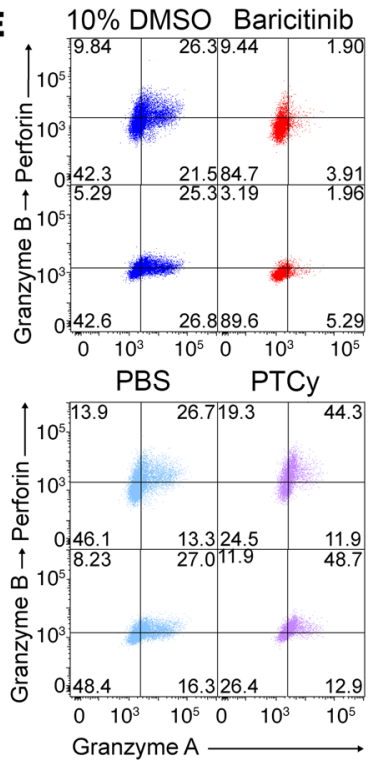

-10\% DMSO $(n=8)$

Baricitinib $(n=8)$

口PBS $(n=8)$
वPTCy $(n=9)$
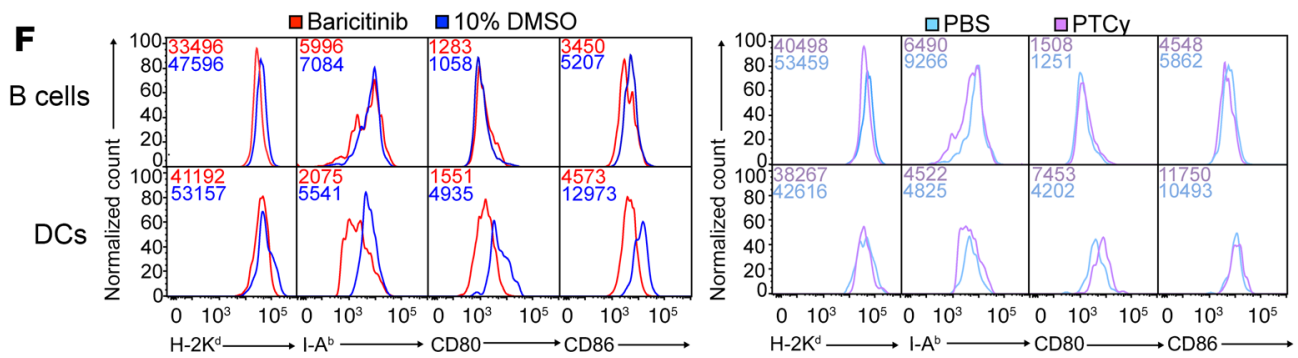

Figure 8. Baricitinib and PTCy curtail pathogenic graft-versus host alloreactivity. (A) Clinical outcomes of irradiated CB6F1 mice infused with B6 splenocytes as per Figure 7 receiving baricitinib or PTCy (red) or their respective vehicle controls (blue). Mice were pooled from 3 experiments; X indicates death o euthanasia. (B) CBCs at 3 weeks after splenocyte infusion; dotted lines represent the lower reference limit. (C) Plasma inflammatory cytokine concentrations 7 days after splenocyte infusion. (D) Absolute donor splenic and bone marrow CD4+ and CD8+ $T$ cell counts and splenic host and donor Treg counts, 7 days after splenocyte infusion. (E and $\mathbf{F}$ ) Splenic donor $C D 8^{+} T$ cell cytolytic enzyme expression (E) and recipient splenic APC phenotyping ( $\mathbf{F}$ ) from mice in $\mathbf{D}$. For $\mathbf{E}$, inset numbers indicate cell frequencies within each quadrant; for $\mathbf{F}$, inset numbers shows MFIs. FACS plots in $\mathbf{E}$ and $\mathbf{F}$ are from 1 representative mouse from 3 experiments. Data points and error bars represent mean \pm SEM. Mantel-Cox test (A, survival), mixed effects model (A, weights and clinical scores), Student's $t$ test (B and $\mathbf{D}$, spleen counts), and Mann-Whitney $U$ test ( $\mathbf{C}$ and $\mathbf{D}$, bone marrow counts) were used for statistical comparisons. ${ }^{*} P<0.05 ;{ }^{* *} P<0.01 ;{ }^{* * *} P<0.001 ;{ }^{* * *} P<0.0001$.

cacy. By simultaneously targeting the stem cell compartment and malignant cells, the therapeutic goals of HSCT can hypothetically be achieved with toxicities largely confined to the hematopoietic system. Indeed, recent work in murine models $(14,15,19,20$, $22)$, nonhuman primates $(52,53)$, and early human trials $(54)$ has demonstrated the feasibility and high efficacy of antibody and ADC-based therapies. Optimization of these strategies for alloHSCT and translation to human clinical trials will benefit from a greater mechanistic understanding of how they modulate donor and recipient immunity, with important implications for treating high-risk malignancies such as AML.

In the present study, we used mouse allo-HSCT models to identify ADC-based conditioning regimens able to achieve robust donor engraftment and to understand the immunobiology underlying their effect. In miHA- and F1-to-parent HSCT, T cell-mediated barriers to engraftment were overcome by combining CD45-SAP with pan-TCD. The transient donor chimerism we often encountered could be explained by incomplete $\mathrm{T}$ cell elimination at all tis- 
A

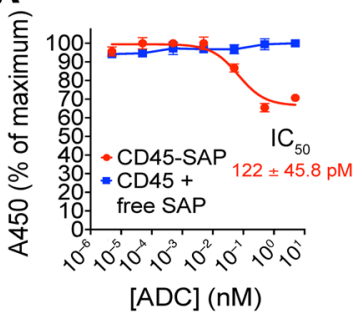

B

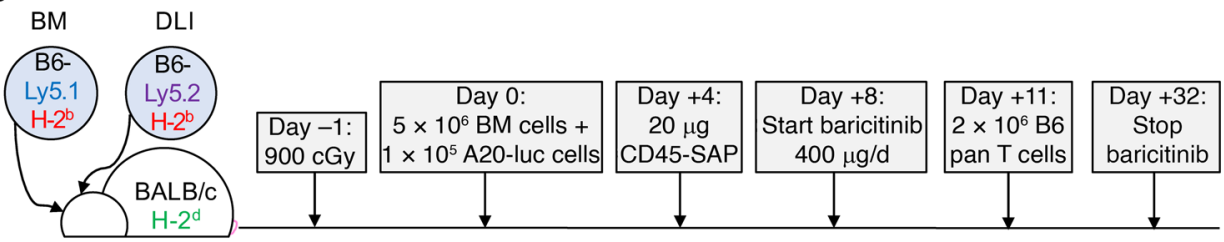

C

No DLI

- TCD BM alone $(n=6)$

- A20 + vehicle $(n=10)$

- A20 + baricitinib $(n=10)$

- A20 + CD45-SAP + vehicle $(n=10)$

\section{DLI day +11}

- TCD BM alone $(n=6)$

- A20 + DLI + vehicle $(n=10)$

$\rightarrow \mathrm{A} 20+\mathrm{DLI}+$ baricitinib

$(n=9)$

- A20 + DLI + CD45-SAP +

vehicle $(n=10)$

A20 + DLI + CD45-SAP +

baricitinib $(n=10)$
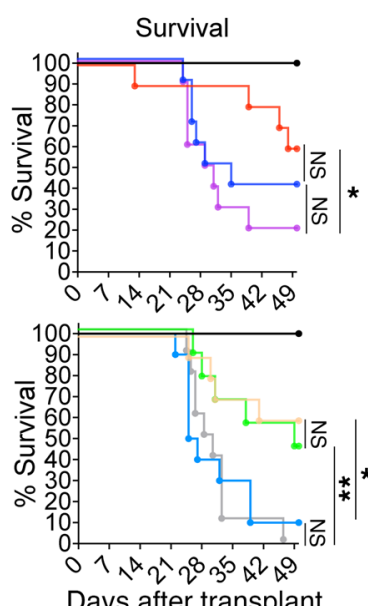

Days after transplant
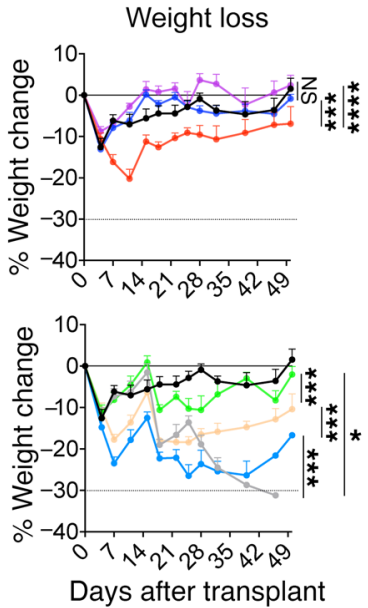

GvHD score
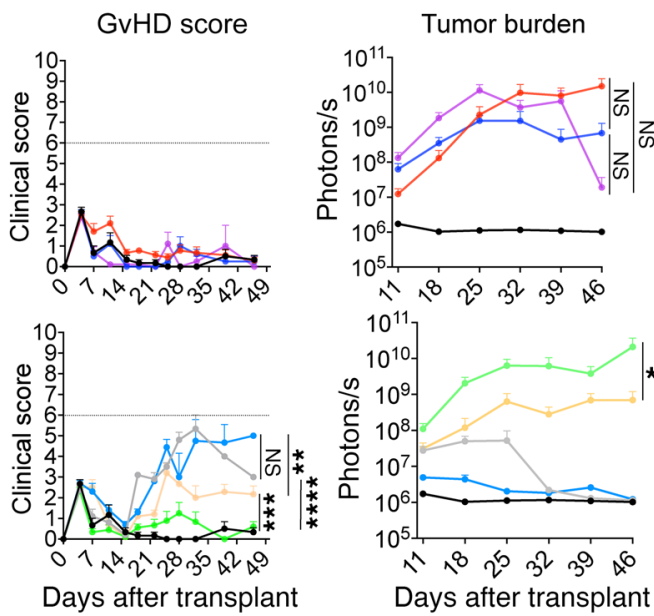

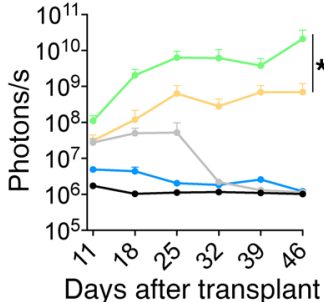

Figure 9. CD45-SAP plus baricitinib balances GvHD and GvL responses. (A) In vitro cytotoxicity of CD45-SAP against luciferase-expressing A20 cells; 1 representative of 3 experiments (6 technical replicates per concentration) is presented. (B) Schematic for delayed DLI model to investigate graftversus-host responses in mice treated with baricitinib and CD45-SAP. (C) Overall survival, weight loss, GvHD outcomes, and tumor burden of mice treated as per B, separated into groups that received or did not receive DLI, pooled from 2 experiments. Data points and error bars represent mean \pm SEM. The Mantel-Cox test (survival) and mixed effects model (weights, clinical scores, and tumor burden) were used for statistical comparisons. ${ }^{*} P<0.05$; ${ }^{* *} P<$ $0.01 ;{ }^{* *} P<0.001 ;{ }^{* * *} P<0.0001$.

sue sites, even with prolonged depleting antibody treatment. Given the high frequency of T cells estimated to be alloreactive (55), even a small residual population of functional host $\mathrm{T}$ cells could reject donor HSCs. To cover alloreactive cells not eliminated by lymphodepleting agents, additional immunosuppressive therapy may be a useful strategy, as we demonstrated by combining daily baricitinib with pre-HSCT TCD. Other more standard immunosuppressants such as PTCy may also be beneficial in this setting; for example, lymphodepletion with antithymocyte globulin combined with PTCy was shown in human trials to mitigate allogeneic $\mathrm{T}$ cell responses, reducing GvHD incidence and nonrelapse mortality (56).

Continuously infused JAK1/2 inhibitors combined with CD45SAP improved upon the shortcomings of pan-TCD, enabling high-level, multilineage engraftment between even fully MHC-mismatched mice without requiring prolonged lymphodepletion. However, the engraftment success rate remained suboptimal, a barrier that was overcome using orally formulated ruxolitinib. While excellent donor chimerism was attainable, a fully myeloablative regimen would further enhance the utility of our approach for the treatment of acute leukemias by maximizing both donor chimerism and antileukemia activity. We are currently pursuing alternative payloads to saporin, particularly small molecule toxins directly conjugated to CD45 and cKit antibodies, to generate fully ablative ADCs. These approaches would avoid issues with indirect conjugates based on streptavidin and saporin, such as immunogenicity and potential interference with endogenous biotin.
We observed notable differences in the effectiveness of ruxolitinib- and baricitinib-based treatment regimens in our studies, but the basis for this is complicated by differences in drug formulation and route of administration. Our PD studies in B6 mice showed ruxolitinib has a considerably shorter duration of effect than baricitinib, possibly underlying the differences in effectiveness when the two compounds are given in equal doses. The dramatic improvement in efficacy seen with the ruxolitinib chow most likely owes to the considerably higher drug doses in this formulation. The drug-delivery method may also affect engraftment success independently of $\mathrm{PD} /$ pharmacokinetic (PK) differences. Of particular concern is the surgical trauma from osmotic pump implantation, which may affect the magnitude of alloresponses via innate immune activation. Oral drug dosing may have improved engraftment outcomes in part by removing this proinflammatory stressor from the peritransplant period. It will be essential in future studies to perform detailed PK studies so that we can harmonize the total drug exposure of ruxolitinib and baricitinib using a common route of administration. This will clarify whether differences in drug efficacy are attributable to PD/PK differences or whether baricitinib and ruxolitinib have unique cellular and molecular effects.

That JAK1/2 inhibition could permit fully MHC-mismatched HSCT demonstrates its ability to overcome both T and NK cellmediated rejection. In vitro and in vivo studies demonstrated a striking effect of baricitinib on NK cell survival and function, which we attributed to blockade of critical signals from IL-15. 
Interestingly, baricitinib selectively inhibited the expansion of alloreactive donor $\mathrm{CD} 8^{+} \mathrm{T}$ cells while sparing $\mathrm{CD} 4^{+} \mathrm{T}$ cells, even expanding donor $\mathrm{CD} 4^{+}$Tregs. $\mathrm{CD}^{+} \mathrm{T}$ cells have been considered the adaptive counterparts of NK cells, given their similar effector functions and transcriptional regulation (57), suggesting there may be important parallels between these cells' responses to JAK1/2 inhibition. One noteworthy effector common to $\mathrm{CD} 8^{+} \mathrm{T}$ cells and NK cells is IFN- $\gamma$, the production of and the response to which were strongly inhibited by baricitinib. Prior studies have highlighted the importance of IFN- $\gamma$ signaling in promoting GvHD and the effect of JAK1/2 inhibition on these signals and the development of disease. Studies with IFN $\gamma \mathrm{R}^{-/-}$mice in an RIC model presented herein also suggest IFN- $\gamma$ signaling influences donor engraftment. Using IFN- $\gamma \mathrm{R}$-deficient donors and recipients, we are investigating whether IFN- $\gamma$ signaling is relevant to how JAK1/2 inhibitors promote engraftment. However, due to the inherent pleiotropy of JAK1/2 inhibition, baricitinib may enhance engraftment via simultaneous modulation of multiple cytokine signaling pathways rather than a single dominant pathway.

Finally, the baricitinib dosing schedule used in this study led to increased pre-DLI A20 tumor burden and worsened tumor control after DLI, which was improved by coadministration of CD45-SAP. The effect of baricitinib in this study contrasts with what we observed in our prior experiments that used a less intensive treatment regimen. As demonstrated with ruxolitinib and baricitinib in our previous studies $(21,58)$, JAK1/2 inhibition enhances tumor growth in vivo when donor $\mathrm{T}$ cells are absent. This could explain the increased pre-DLI tumor burden (on $\mathrm{d}+11$ ) in mice that started baricitinib 3 days in advance of DLI (on d+8). An increased pre-DLI A20 tumor burden would unfavorably shift the effector/target ratio, thus compromising the ability of donor $\mathrm{T}$ cells to restrain A20 cell expansion.

By inhibiting donor $\mathrm{CD}^{+} \mathrm{T}$ cells and reducing the costimulatory function of host APCs, it is not unexpected that JAK1/2 inhibition would interfere with both GvHD and GvL responses. However, the results of our current and prior studies suggest the intriguing possibility that the effects of JAK1/2 inhibition on beneficial and harmful graft-versus-host responses can be uncoupled by fine-tuning the drug-dosing schedule. Studies are already underway to better understand how alterations in baricitinib dosing affect the development of GvHD and GvL (59) and the mechanistic basis for this behavior.

Mice receiving CD45-SAP consistently showed lower pre-DLI tumor burdens, indicative of a direct antitumor effect that aided control of A20 proliferation by DLI. That the addition of CD45SAP somewhat worsened GvHD in DLI mice receiving baricitinib suggested that the ADC enhanced the donor alloresponse, thereby indirectly contributing to antitumor activity. We noted in early studies that sAV-SAP alone increased granulocyte counts in B6 mice, (Supplemental Figure 1), which may reflect a mild inflammatory response to sAV-SAP treatment able to augment donor T cell responses. However, any inflammation induced by the ADCs alone was not sufficient to promote pathogenic graft-versus-host alloreactivity in our parent-to-F1 model (Figure 7). The impact of CD45SAP on donor alloresponses may have been more prominent in our GvL experiments due to the combined inflammatory insult of $\mathrm{ADC}$ infusion 4 days after lethal irradiation or strain differences in responses to sAV-SAP between CB6F1 and BALB/c mice.
In conclusion, the studies presented herein exemplify the promise of immunotherapy to provide safe, effective conditioning for HSCT. Importantly, our studies provide insights into the unique immunobiology of ADC-conditioned allo-HSCT and an experimental foundation on which further basic and translational investigations can be conducted.

\section{Methods}

Mice. The following strains were purchased from Jackson Laboratories: C57BL6/J (JAX 000664), BALB/cJ (stock no. 000651), DBA/2J (stock no. 000671), CB6F1/J (stock no. 100007), C57BL/6-Tg(UBCGFP)30Scha/J (B6-GFP; stock no. 004353), and CByJ.SJL(B6)-

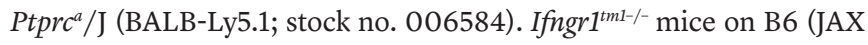
003288) and BALB/c (JAX 007077) backgrounds were provided by Herbert Virgin (Department of Pathology and Immunology, Washington University) and were bred in-house. All mice were maintained in specific pathogen-free colonies and received ad libitum water and standard chow (LabDiet 5053; Lab Supply). Age- and sex-matched mice 6 to 12 weeks of age were used for all experiments and assigned randomly to treatment groups. All lethally irradiated mice received trimethoprim-sulfamethoxazole (SulfaTrim, $5 \mathrm{~mL}$ per $250 \mathrm{~mL}$ drinking water) for 2 weeks after irradiation; ADC-conditioned or sublethally irradiated mice did not receive antibiotic prophylaxis. For retroorbital injections, mice were anesthetized with $3 \%$ isoflurane in $\mathrm{O}_{2}$. For survival surgeries, mice were anesthetized via i.p. injection of $80-100$ $\mathrm{mg} / \mathrm{kg}$ ketamine plus 5 to $10 \mathrm{mg} / \mathrm{kg}$ xylazine. Surgical sites were shaved, disinfected, and draped in sterile fashion prior to first incision; skin closure was done using $9 \mathrm{~mm}$ autoclips and buprenorphine (0.1 $\mathrm{mg} / \mathrm{kg}$ ) provided for postoperative analgesia.

Mouse tissue preparation. Spleen, lymph node, and thymus singlecell suspensions were prepared by gentle homogenization with a syringe plunger through a $70 \mu \mathrm{m}$ filter in PBS containing 0.5\% BSA and 2 mM EDTA (running buffer). Bone marrow from femurs and tibias was harvested by centrifugation $(10,000 \mathrm{~g}$ for 10 seconds) as previously described (60). Mouse peripheral blood was drawn from the facial vein using Goldenrod $5 \mathrm{~mm}$ animal lancets (Medipoint) and collected into $\mathrm{K}_{2}$ EDTA-coated tubes (BD). Erythrocytes were removed by ammonium chloride-potassium bicarbonate (ACK) lysis.

Cell culture and in vitro assays. R10 medium was prepared by combining RPMI (Gibco, Thermo Fisher Scientific) with 10\% FBS (R\&D Systems), GlutaMAX (Gibco, Thermo Fisher Scientific), and penicillin/streptomycin (Gibco, Thermo Fisher Scientific). K10 medium was prepared by supplementing R10 with $10 \mathrm{mM}$ HEPES, $0.1 \mathrm{mM}$ nonessential amino acids, $1 \mathrm{mM}$ sodium pyruvate, and $55 \mu \mathrm{M} 2$-mercaptoethanol. Human PBMCs were harvested from leukoreduction chambers by Ficoll density gradient centrifugation (1000 $g$ for 20 minutes) and cryopreserved. The A2O and YAC- 1 cell lines were obtained from ATCC, tested negative for Mycoplasma, and maintained in R10 medium. The XTT Viability Assay Kit was provided by Cell Signaling Technologies and performed per the manufacturer's protocol. All cultures were incubated at $37^{\circ} \mathrm{C} / 5 \% \mathrm{CO}_{2}$.

For ex vivo stimulation of mouse $\mathrm{T}$ cells, $1 \times 10^{5} \mathrm{~T}$ cells purified from spleen and lymph nodes with the EasySep Mouse $\mathrm{T}$ cell Isolation Kit (StemCell Technologies) were cultured with $4 \times 10^{5}$ T-depleted splenocytes in 96-well round-bottom plates in R10 medium plus $1 \mu \mathrm{g} / \mathrm{mL} \alpha \mathrm{CD} 3$. Supernatants were analyzed for cytokines after 24 hours and cells analyzed for expansion by flow cytometry at 
72 hours. Cell labeling with $5 \mu \mathrm{M}$ CFSE was done per the manufacturer's protocol (BioLegend).

For mouse NK cell assays, splenic NK cells were enriched to more than $80 \%$ purity using the EasySep Mouse NK Cell Isolation Kit (StemCell Technologies), and 1.0 to $2.5 \times 10^{4} \mathrm{NK}$ cells per well were cultured in K10 medium plus IL-15 alone (1-100 ng/mL) or a cocktail of $10 \mathrm{ng} / \mathrm{mL} \mathrm{IL-12,} 10 \mathrm{ng} / \mathrm{mL}$ IL-15, and $50 \mathrm{ng} / \mathrm{mL}$ IL-18 (all murine cytokines; BioLegend; ref. 33). For cytotoxicity assays, purified NK cells were stimulated with $100 \mathrm{ng} / \mathrm{mL}$ IL-15 for 48 hours, washed twice to remove cytokines, then replated for 4 hours at various effector-to-target (E:T) ratios in a 96-well, round-bottom plate with YAC-1 target cells (2000 cells/well). YAC-1 cell death was assessed with Zombie viability dyes (BioLegend).

For CFU assays, whole murine bone marrow was incubated in complete methylcellulose medium (R\&D Systems) per the manufacturer's protocol $\left(2 \times 10^{4}\right.$ cells/plate for 12 days $)$.

$C B C$. WBC, hematocrit, and platelet counts were obtained with a Hemavet 950 analyzer (Drew Scientific). Reference ranges were as follows: WBCs, $1.8-10.7 \times 10^{3}$ cells $/ \mu \mathrm{L}$; hematocrit, $35.1 \%-45.4 \%$; platelets, $592-2,972 \times 10^{3}$ cells $/ \mu \mathrm{L}$. In engraftment experiments, CBCs from age- and sex-matched cohorts of untreated B6 $(n=16)$ and DBA/2 $(n=$ 19) mice were used for the pre-HSCT ( $t=0$ months) time point.

Flow cytometry. Flow cytometry was performed with a Beckman Coulter Gallios instrument equipped with Kaluza acquisition software; data analysis was done using Treestar FlowJo, version 10.7. Supplemental Table 1 lists all antibodies and flow cytometry reagents. For labeling of fresh, unfixed samples, single-cell suspensions were incubated with antibodies in $100 \mu \mathrm{L}$ running buffer for 15 to $20 \mathrm{~min}$ utes. For viability staining, $1 \mu \mathrm{g} / \mathrm{mL}$ 7-aminoactinomycin D (7-AAD) (BioLegend) was added immediately before analysis. For intracellular cytokine and cytotoxic enzyme staining, cells were stained with Zombie viability dye in protein-free PBS for 15 minutes, stained 15 minutes for surface markers, then fixed for 20 minutes with $4 \%$ paraformaldehyde (PFA) in PBS (BioLegend). Fixed cells were then permeabilized with $0.5 \%$ saponin in running buffer and stained 30 minutes for intracellular markers. FoxP3 staining was done using the FoxP3/Transcription Factor Staining Buffer Set per the manufacturer's instructions (eBioscience). All staining steps were performed at $20^{\circ} \mathrm{C}$. Gating strategies for HSPC analysis were as described previously (15).

Phosphoflow analysis. For phospho-Stat1 analysis, whole blood was stimulated for 15 minutes with $100 \mathrm{ng} / \mathrm{mL}$ murine $\mathrm{IFN}-\gamma$ at $37^{\circ} \mathrm{C}$, then immediately fixed with $1 \mathrm{~mL}$ Lyse/Fix Buffer (BD) for 10 minutes at $37^{\circ} \mathrm{C}$. For phospho-Stat 3 analysis, cryopreserved human PBMCs were thawed and rested overnight at $37^{\circ} \mathrm{C}$ in R10, stimulated with $100 \mathrm{ng} /$ $\mathrm{mL}$ human IL-6 for 15 minutes at $37^{\circ} \mathrm{C}$, then fixed in $4 \%$ PFA in PBS. For phospho-Stat 5 analysis, purified $\mathrm{B} 6$ mouse splenic NK cells were incubated for 30 minutes with $100 \mathrm{ng} / \mathrm{mL}$ IL-15 in K10 medium, then fixed in $4 \%$ PFA.

After stimulation and fixation, all samples were permeabilized in ice-cold Perm Buffer III (BD) and held at $-20^{\circ} \mathrm{C}$ overnight. Samples were then washed 3 times with running buffer and stained at $20^{\circ} \mathrm{C}$ for phospho-Stat molecules (all 1-hour incubations). For phospho-Stat1 (Y701), samples were incubated with primary rabbit anti-phospho Stat1 (Cell Signaling Technologies, clone 58D6), washed, then stained with Alexa Fluor 647-conjugated polyclonal anti-rabbit secondary antibody (Cell Signaling Technologies, catalog 4414). For phospho-Stat3 (Y705), samples were incubated with anti-human CD4 (BioLegend, clone RPA-T4) and anti-phospho Stat3 (BioLegend, clone 13A3-1). For phospho-Stat5 (Y694), samples were incubated with anti-NK1.1 (BioLegend, clone PK136) and anti-phospho Stat5 (BD, clone 47).

Cytokine analysis. Cytokine concentrations in culture supernatant or mouse plasma were measured with the LegendPLEX Inflammation Panel (13-plex) or the Mouse Th1 Panel (5-plex) per the manufacturer's protocols (BioLegend). Quantification was done using LegendPLEX software, version 8.0. For statistics, samples whose cytokine concentration was too low to quantify were assigned the value of the lower limit of quantitation. For intracellular IFN- $\gamma$ analysis of ex vivostimulated splenic NK cells, cells were cultured in K1O medium with or without cytokine stimulation for 15 hours; then $5 \mu \mathrm{g} / \mathrm{mL}$ Brefeldin A (BioLegend) was added for the last 2.5 hours. Cells were then fixed, saponin permeabilized, and stained.

In vivo NK cell activation. Poly I:C (Invivogen) was injected (100 $\mu$ i.p.) as previously described (35). Splenocytes were harvested 4 hours later, immediately incubated in K10 medium plus Brefeldin A for 4 hours, then stained for intracellular cytokines. Cytolytic enzyme staining and cytotoxicity assays were performed with splenocytes harvested 24 hours after Poly I:C injection. For cytotoxicity assays, total splenic NK cells were enumerated, and whole splenocytes were plated with YAC-1 target cells to achieve 5:1 and 1:1 NK/YAC-1 ratios.

Preparation of saporin ADCs. sAV-SAP (average saporin/streptavidin ratio of 2.4) was obtained from Advanced Targeting Systems. A total molecular weight of $287 \mathrm{kDa}(127 \mathrm{kDa}$ for sAV-SAP + $160 \mathrm{kDa}$ for IgG) was used for ADC dose calculations. To prepare ADCs, biotinylated CD45.2 (clone 104, BioLegend) and c-Kit (clone 2B8, BioLegend) antibodies were incubated with SAV-SAP (1:1 molar ratio) for 15 minutes at $20^{\circ} \mathrm{C}$. CD 45 - and cKit-SAP were then diluted to their final concentration in endotoxin-free PBS (MilliporeSigma) and injected retroorbitally. Sodium azide and endotoxin were removed from antibodies with Zeba desalting spin columns and High-Capacity Endotoxin Removal spin columns (Thermo Fisher) per the manufacturer's instructions, then sterilized using a $0.22 \mu \mathrm{m}$ PES syringe filter.

For experiments in which free antibody and free sAV-SAP were administered together, noninteraction was ensured by using nonbiotinylated antibodies and SAV-SAP whose biotin-binding sites were occupied by an irrelevant biotinylated peptide (BLANK Streptavidin-SAP). For experiments in which free antibody or sAV-SAP were administered alone, the equivalent mass of each component alone in the ADC was administered to each mouse (i.e., the doses of CD45.2 antibody and sAV-SAP corresponding to $75 \mu \mathrm{g}$ CD 45 -SAP are $41.8 \mu \mathrm{g}$ and $33.2 \mu$ g, respectively). To avoid interference by cKit-SAP, bone marrow flow cytometry was done using c-Kit clone ACK2.

HSCT with ADC conditioning. Mice were injected with CD45-SAP or cKit-SAP 7 days before HSCT (d-7). On transplant day (d0), mice were injected retroorbitally with 10 to $20 \times 10^{6}$ whole donor bone marrow cells. For serial transplantation, mice received a single lethal irradiation dose (1100 cGy for B6 mice, 950 cGy for DBA/2 mice) from a Mark I Model 30 irradiator (J.L. Shepherd and Associates, ${ }^{137} \mathrm{Cs}$ source, $73.69 \mathrm{cGy} / \mathrm{min}$ ) and transplanted with $10 \times 10^{6}$ whole bone marrow cells from primary transplant recipients 8 to 16 hours after irradiation.

In vivo lymphocyte depletion. Depleting antibodies and isotype controls (InVivoPlus Grade) were obtained from BioXcell. CD4 ${ }^{+}$and CD8 ${ }^{+}$ T cells were depleted using clones GK1.5 and YTS169.4, respectively, and NK cells depleted using $\alpha$ NK1.1 clone PK136. Mouse IgG2aк (clone

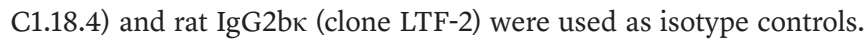


All antibodies were administered i.p. at $250 \mu \mathrm{g}$ per dose. Cell depletion was confirmed by FACS; to avoid interference with depleting antibodies, $\mathrm{CD} 4^{+} \mathrm{T}$ cells were stained with clone $\mathrm{RM} 4-4, \mathrm{CD} 8^{+} \mathrm{T}$ cells with clone 53-6.7, and NK cells with CD3, CD49b (DX5), and NKp46.

Daily drug treatments. The selective JAK1/2 inhibitors baricitinib (LY3009104, INCB028050) and ruxolitinib (INCB18424) were obtained from MedChemExpress. For s.c. administration, baricitinib was dissolved in $100 \% \mathrm{DMSO}$ at $20 \mathrm{mg} / \mathrm{mL}$ and stored at $-20^{\circ} \mathrm{C}$. These DMSO stocks were thawed and diluted 1:10 in PBS; $200 \mu \mathrm{L} /$ mouse was injected s.c. immediately $(400 \mu \mathrm{g})$. PBS+10\% DMSO was used as the vehicle control.

Cyclophosphamide hydrate (Cayman Chemical) was dissolved in PBS at $25 \mathrm{mg} / \mathrm{mL}$ and stored at $-20^{\circ} \mathrm{C}$. These stocks were diluted $1: 8$ in PBS and injected i.p. at $200 \mu \mathrm{L} /$ mouse ( $625 \mu \mathrm{g}$ daily, $25 \mathrm{mg} / \mathrm{kg})$ on $\mathrm{d}+3$ and $\mathrm{d}+4$ relative to HSCT or splenocyte infusion.

Continuous administration of JAK inhibitors. ALZET subcutaneous osmotic pumps (model 2004) delivered JAK1/2 inhibitors continuously for 28 days at $6 \mu \mathrm{L} / \mathrm{d}$ in a vehicle of $50 \% \mathrm{DMSO} / 50 \%$ polyethylene glycol 400 (PEG-400). JAK1/2 inhibitors were prepared at $2 \times$ concentration $(133.3 \mathrm{mg} / \mathrm{mL})$ in $100 \%$ DMSO, then diluted to $1 \times$ with PEG$400(66.7 \mathrm{mg} / \mathrm{mL}, 400 \mu \mathrm{g}$ total daily dose). Compounds were loaded into osmotic pumps and surgically implanted per the manufacturer's instructions. Ruxolitinib-containing mouse chow (2 g drug/kg chow; Incyte) was administered ad libitum; per the manufacturer, steadystate ruxolitinib plasma concentrations of 600-1200 nM are expected by 3 days after the chow is started.

Skin grafting. Skin implantation was performed as described (61). Briefly, donor ear skin from BALB/CJ and DBA/2J mice was harvested and held in ice-cold PBS prior to transplant. Skin recipients were DBA/2J mice that either successfully engrafted with BALB-Ly5.1 bone marrow (BALB-DBA mixed chimeras) or those that failed to engraft. Recipients were prepared for survival surgery, and a small patch of dorsal skin was resected and replaced with donor skin. Recipients were then bandaged, single-housed, and monitored for 4 days to ensure the graft bed remained undisturbed. Bandages were then removed, and recipients monitored daily for graft viability.

Graft-versus-host alloreactivity model and in vivo mixed leukocyte reactions. A parent-to- $\mathrm{F} 1$ adoptive transfer model was used to study $\mathrm{T}$ cell alloreactivity in vivo, as previously described (50). CB6F1 recipients were conditioned with CD45-SAP 7 days before adoptive transfer or with $500 \mathrm{cGy}$ irradiation 8 to 16 hours before adoptive transfer, then infused with $25 \times 10^{6} \mathrm{~B} 6$ splenocytes. Irradiated recipients infused with CB6F1 (syngeneic) splenocytes and unconditioned recipients infused with B6 splenocytes were used as negative controls. Clinical scoring of mice was done based on a 10-point scale ( $0-2$ points each for posture, activity, fur ruffling, weight loss, and skin lesions) (62). For in vivo mixed leukocyte reactions, recipient mice were infused with 2 to $3 \times 10^{6} \mathrm{CFSE}-$ labeled donor $\mathrm{T}$ cells, and CFSE dilution was assessed in recipient spleens 72 hours later.

GvL model. GvL studies were performed using a delayed DLI model with bioluminescent imaging as described (21). Briefly, BALB/c recipients were lethally irradiated (900 cGy) on $\mathrm{d}-1$, then engrafted on d0 with $1 \times 10^{5}$ luciferase-expressing A20 lymphoma cells plus $5 \times$ $10^{6} \mathrm{~T}$ cell-depleted bone marrow cells from B6-CD45.1 mice. Recipients were then infused with $2 \times 10^{6} \mathrm{~B} 6$ pan T cells on $\mathrm{d}+11$. CD 45-SAP was administered in a single $20 \mu \mathrm{g}$ dose on $\mathrm{d}+4$ (7 days before DLI); this lower CD45-SAP dose was used because irradiated BALB/c mice poorly tolerated the full $75 \mu \mathrm{g}$ CD $45-S A P$ dose. Baricitinib was administered daily from $\mathrm{d}+8$ to $\mathrm{d}+32(400 \mu \mathrm{g} \times 25$ doses $)$.

Histopathology. Femurs were preserved in neutral buffered formalin (PBS plus 3.7\% formaldehyde) and incubated at $20^{\circ} \mathrm{C}$ with gentle rocking for at least 48 hours. Decalcification, preparation of formalin-fixed paraffin-embedded sections, and H\&E staining were performed by the Washington University Department of Comparative Medicine Animal Diagnostic Laboratory.

Statistics. Sample size determinations and analysis parameters were based on general guidelines for animal research (63). Statistical analysis was done using GraphPad Prism 9. IC $_{50}$ values for cytotoxicity studies were derived from curve-fitting dose-response data to a 3-variable inhibition model. The Shapiro-Wilk test was used to assess data normality. For comparison of 2 data sets, normally distributed data were analyzed with unpaired, 2-tailed Student's $t$ tests with Welch's correction (no assumption of equal variance); nonnormally distributed data were analyzed with the Mann-Whitney $U$ test. For comparisons of multiple groups with a single common control group, 1-way ANOVA was used for normally distributed data (corrected for multiple comparisons with Dunnett's test) and the Kruskal-Wallis test used for nonnormally distributed data (corrected for multiple comparisons with Dunn's test). CBC values were compared with the lower reference limit using a 1-sample $t$ test. Survival analysis was done with the Mantel-Cox log-rank test. Time-course experiments (donor chimerism, weight changes, clinical scoring, and tumor burden) were analyzed with 2-way repeated measures ANOVA; a mixed effects model for repeated measures (maximum likelihood method) was used instead if data were missing due to mouse deaths. The criterion for statistical significance was $P<0.05$.

Study approval. Mice were humanely handled in accordance with an animal protocol approved by the Institutional Animal Care and Use Committee at Washington University School of Medicine.

\section{Author contributions}

SPP, PGR, MLC, MPR, JC, and JFD designed research. SPP, JKR, SK, SL, and JC conducted experiments. SPP, SK, SL, and JC analyzed data. SPP and JFD wrote the manuscript.

\section{Acknowledgments}

This study was funded by $\mathrm{NIH} /$ National Cancer Institute (NCI) R35CA210084 (to JFD), NIH/NCI Leukemia SPORE grant (P50CA171963 to JFD) and Leukemia SPORE Career Enhancement Award (P50CA171063 to SPP), an American Society for Transplantation and Cellular Therapy (ASTCT) New Investigator Award (to SPP), an NCI Research Specialist Award (R50CA211466 to MPR), a donation from Bigelow Aerospace (to JFD), awards from Gabrielle's Angel Foundation for Cancer Research (to SPP), a grant from the Be the Match Foundation Amy Strelzer Manasevit Research Program (to JC), and funds from the Siteman Cancer Center Investment Program (supported by the Foundation for Barnes-Jewish Hospital Cancer Frontier Fund, NCI Cancer Center Support Grant, P30CA091842, and Barnard Trust to JC). B6 mice for in vitro T and NK cell assays were a kind gift from P.M. Allen. We thank P.M. Allen, J. Eissenberg, and L. Eissenberg for review and discussion of the manuscript.

Address correspondence to: John DiPersio, Campus Box 8007, 660 South Euclid Ave., St. Louis, Missouri 63110, USA. Phone: 314.454.8306; Email: jdipersi@wustl.edu. 
1. Duarte RF, et al. Indications for haematopoietic stem cell transplantation for haematological diseases, solid tumours and immune disorders: current practice in Europe, 2019. Bone Marrow Transplant. 2019;54(10):1525-1552.

2. Gupta RK, et al. HIV-1 remission following CCR5 $\triangle 32 / \Delta 32$ haematopoietic stem-cell transplantation. Nature. 2019;568(7751):244-248.

3. Sykes M. Transplantation: moving to the next level. Immunol Rev. 2014;258(1):5-11.

4. Perkey E, Maillard I. New insights into graftversus-host disease and graft rejection. Annu Rev Pathol. 2018;13:219-245.

5. Gyurkocza B, Sandmaier BM. Conditioning regimens for hematopoietic cell transplantation: one size does not fit all. Blood. 2014;124(3):344-353.

6. Czechowicz A, Weissman IL. Purified hematopoietic stem cell transplantation: the next generation of blood and immune replacement. Hematol Oncol Clin North Am. 2011;25(1):75-87.

7. Dickinson AM, et al. Graft-versus-leukemia effect following hematopoietic stem cell transplantation for leukemia. Front Immunol. 2017;8:496.

8. Scott BL, et al. Myeloablative versus reducedintensity hematopoietic cell transplantation for acute myeloid leukemia and myelodysplastic syndromes. JClin Oncol. 2017;35(11):1154-1161.

9. National Cancer Institute. Cancer Stat Facts: Leukemia - Acute Myeloid Leukemia (AML). https://seer.cancer.gov/statfacts/html/amyl. html. Updated September 27, 2021. Accessed October 14, 2021.

10. Almeida AM, Ramos F. Acute myeloid leukemia in the older adults. Leuk Res Rep. 2016;6:1-7.

11. Armand P, et al. Impact of cytogenetics on outcome of de novo and therapy-related AML and MDS after allogeneic transplantation. Biol Blood Marrow Transplant. 2007;13(6):655-664.

12. Byrd JC, et al. Pretreatment cytogenetic abnormalities are predictive of induction success, cumulative incidence of relapse, and overall survival in adult patients with de novo acute myeloid leukemia: results from Cancer and Leukemia Group B (CALGB 8461). Blood. 2002;100(13):4325-4336.

13. Ossenkoppele G, Lowenberg B. How I treat the older patient with acute myeloid leukemia. Blood. 2015;125(5):767-774.

14. Palchaudhuri R, et al. Non-genotoxic conditioning for hematopoietic stem cell transplantation using a hematopoietic-cell-specific internalizing immunotoxin. Nat Biotechnol. 2016;34(7):738-745.

15. Czechowicz A, et al. Selective hematopoietic stem cell ablation using CD117-antibody-drug-conjugates enables safe and effective transplantation with immunity preservation. Nat Commun. 2019;10(1):617.

16. Gao C, et al. Nongenotoxic antibody-drug conjugate conditioning enables safe and effective platelet gene therapy of hemophilia A mice. Blood Adv. 2019;3(18):2700-2711.

17. Srikanthan MA, et al. Effective multi-lineage engraftment in a mouse model of fanconi anemia using non-genotoxic antibody-based conditioning. Mol Ther Methods Clin Dev. 2020;17:455-464.

18. Castiello MC, et al. Efficacy and safety of anti-CD45-saporin as conditioning agent for RAG deficiency. JAllergy Clin Immunol.
2021;147(1):309-320.

19. George BM, et al. Antibody conditioning enables mhc-mismatched hematopoietic stem cell transplants and organ graft tolerance. Cell Stem Cell. 2019;25(2):185-192.

20. Li Z, et al. Hematopoietic chimerism and donor-specific skin allograft tolerance after non-genotoxic CD117 antibody-drug-conjugate conditioning in MHC-mismatched allotransplantation. Nat Commun. 2019;10(1):616.

21. Choi J, et al. Baricitinib-induced blockade of interferon gamma receptor and interleukin-6 receptor for the prevention and treatment of graft-versus-host disease. Leukemia. 2018;32(11):2483-2494.

22. Chhabra A, et al. Hematopoietic stem cell transplantation in immunocompetent hosts without radiation or chemotherapy. Sci Transl Med. 2016;8(351):351ra105.

23. Wachsmuth LP, et al. Optimized timing of post-transplantation cyclophosphamide in MHC-haploidentical murine hematopoietic cell transplantation. Biol Blood Marrow Transplant. 2020;26(2):230-241.

24. Gavegnano C, et al. Baricitinib reverses HIVassociated neurocognitive disorders in a SCID mouse model and reservoir seeding in vitro. J Neuroinflammation. 2019;16(1):182.

25. Kleppe M, et al. Jak1 integrates cytokine sensing to regulate hematopoietic stem cell function and stress hematopoiesis. Cell Stem Cell. 2017;21(4):489-501.

26. Neubauer $\mathrm{H}$, et al. Jak2 deficiency defines an essential developmental checkpoint in definitive hematopoiesis. Cell. 1998;93(3):397-409.

27. Rodig SJ, et al. Disruption of the Jak1 gene demonstrates obligatory and nonredundant roles of the Jaks in cytokine-induced biologic responses. Cell. 1998;93(3):373-383.

28. Bottos A, et al. Decreased NK-cell tumour immunosurveillance consequent to JAK inhibition enhances metastasis in breast cancer models. Nat Commun. 2016;7:12258.

29. Schonberg K, et al. JAK inhibition impairs NK cell function in myeloproliferative neoplasms. Cancer Res. 2015;75(11):2187-2199.

30. Ritchie DS, et al. Venetoclax or ruxolitinib depletion of recipient NK cells, in combination with reduced intensity conditioning, improves donor cell engraftment without GVHD in a mouse model of allosct. Biol Blood Marrow Transplant. 2020;26(3):S171.

31. Beilke JN, et al. The requirement for NKG2D in NK cell-mediated rejection of parental bone marrow grafts is determined by MHC class I expressed by the graft recipient. Blood. 2010;116(24):5208-5216.

32. Gotthardt D, et al. JAK/STAT cytokine signaling at the crossroad of NK cell development and maturation. Front Immunol. 2019;10:2590.

33. Cooper MA, et al. Cytokine-induced memorylike natural killer cells. Proc Natl Acad Sci U S A. 2009;106(6):1915-1919.

34. Fehniger TA, et al. Acquisition of murine NK cell cytotoxicity requires the translation of a preexisting pool of granzyme $B$ and perforin mRNAs. Immunity. 2007;26(6):798-811.

35. McCartney S, et al. Distinct and complementary functions of MDA5 and TLR3 in poly(I:C)mediated activation of mouse NK cells. J Exp Med. 2009;206(13):2967-2976.

36. Dolai S, et al. Quantitative phosphotyrosine profiling of patient-derived xenografts identifies therapeutic targets in pediatric leukemia. Cancer Res. 2016;76(9):2766-2777.

37. Han ES, et al. Ruxolitinib synergistically enhances the anti-tumor activity of paclitaxel in human ovarian cancer. Oncotarget. 2018;9(36):24304-24319.

38. Iacobucci I, et al. Truncating erythropoietin receptor rearrangements in acute lymphoblastic leukemia. Cancer Cell. 2016;29(2):186-200.

39. Maude SL, et al. Efficacy of JAK/STAT pathway inhibition in murine xenograft models of early T-cell precursor (ETP) acute lymphoblastic leukemia. Blood. 2015;125(11):1759-1767.

40. Ollila S, et al. Stromal Lkb1 deficiency leads to gastrointestinal tumorigenesis involving the IL-11-JAK/STAT3 pathway. J Clin Invest. 2018;128(1):402-414.

41. Taniguchi K, et al. YAP-IL-6ST autoregulatory loop activated on APC loss controls colonic tumorigenesis. Proc Natl Acad Sci U S A. 2017;114(7):1643-1648

42. Afram G, et al. Reduced intensity conditioning increases risk of severe cGVHD: identification of risk factors for cGVHD in a multicenter setting. Med Oncol. 2018;35(6):79.

43. Im A, et al. Risk factors for graft-versus-host disease in haploidentical hematopoietic cell transplantation using post-transplant cyclophosphamide. Biol Blood Marrow Transplant. 2020;26(8):1459-1468.

44. Levine JE, et al. Lowered-intensity preparative regimen for allogeneic stem cell transplantation delays acute graft-versus-host disease but does not improve outcome for advanced hematologic malignancy. Biol Blood Marrow Transplant. 2003;9(3):189-197.

45. Mielcarek M, et al. Graft-versus-host disease after nonmyeloablative versus conventional hematopoietic stem cell transplantation. Blood. 2003;102(2):756-762.

46. Nakasone H, et al. Impact of conditioning intensity and TBI on acute GVHD after hematopoietic cell transplantation. Bone Marrow Transplant. 2015;50(4):559-565.

47. Henden AS, Hill GR. Cytokines in graft-versus-host disease. JImmunol. 2015;194(10):4604-4612.

48. Schroeder MA, DiPersio JF. Mouse models of graft-versus-host disease: advances and limitations. Dis Model Mech. 2011;4(3):318-333.

49. Toubai T, et al. Danger signals and graft-versushost disease: current understanding and future perspectives. Front Immunol. 2016;7:539.

50. Yada S, et al. The role of $\mathrm{p} 53$ and Fas in a model of acute murine graft-versus-host disease. JImmunol. 2005;174(3):1291-1297.

51. Jagasia $\mathrm{M}$, et al. Ruxolitinib for the treatment of steroid-refractory acute GVHD (REACH1): a multicenter, open-label phase 2 trial. Blood. 2020;135(20):1739-1749.

52. Kwon HS, et al. Anti-human CD117 antibodymediated bone marrow niche clearance in nonhuman primates and humanized NSG mice. Blood. 2019;133(19):2104-2108.

53. Tisdale JF, et al. A single dose of CD117 antibody 


\section{RESEARCH ARTICLE}

drug conjugate enables autologous genemodified hematopoietic stem cell transplant (gene therapy) in nonhuman primates. Blood. 2019;134(suppl_1):610.

54. Agarwal R, et al. Toxicity-free hematopoietic stem cell engraftment achieved with anti-CD117 monoclonal antibody conditioning. Biol Blood Marrow Transplant. 2019;25(3):S92.

55. Morris GP, et al. Alloreactivity is limited by the endogenous peptide repertoire. Proc Natl Acad Sci U S A. 2011;108(9):3695-3700.

56. Pasic I, et al. Post-transplant cyclophosphamide combined with anti-thymocyte globulin for graftvs-host disease prophylaxis improves survival and lowers non-relapse mortality in older patients undergoing allogeneic hematopoietic cell transplantation. Ann Hematol. 2020;99(6):1377-1387.

57. Kurioka A, et al. Innate-like CD8+ T-cells and NK cells: converging functions and phenotypes. Immunology. 2018;154(4):547-556.

58. Choi J, et al. Pharmacologic blockade of JAK1/JAK2 reduces GvHD and preserves the graft-versus-leukemia effect. PLoS One. 2014;9(10):e109799.

59. Kim S, et al. Baricitinib prevents GvHD by increasing Tregs via JAK3 and treats established GvHD by promoting intestinal tissue repair via EGFR [published online July 24, 2021]. Leukemia.
The Journal of Clinical Investigation

https://doi.org/10.1038/s41375-021-01360-9.

60. Amend SR, et al. Murine hind limb long bone dissection and bone marrow isolation. J Vis Exp. 2016(110):53936.

61. Cheng CH, et al. Murine full-thickness skin transplantation. J Vis Exp. 2017(119):55105.

62. Cooke KR, et al. An experimental model of idiopathic pneumonia syndrome after bone marrow transplantation: $\mathrm{I}$. The roles of minor $\mathrm{H}$ antigens and endotoxin. Blood. 1996;88(8):3230-3239.

63. Festing MF, Altman DG. Guidelines for the design and statistical analysis of experiments using laboratory animals. ILAR J. 2002;43(4):244-258. 